\title{
$120 \mathrm{GeV}$ neutrino physics graphite target damage assessment using electron microscopy and high-energy x-ray diffraction
}

\author{
N. Simos, ${ }^{1,4}$ P. Hurh, ${ }^{2}$ E. Dooryhee, ${ }^{1}$ L. Snead, ${ }^{4}$ D. Sprouster, ${ }^{4}$ Z. Zhong, ${ }^{1}$ H. Zhong, ${ }^{4}$ S. Ghose,${ }^{1}$ \\ Z. Kotsina,${ }^{5}$ K. Ammigan, ${ }^{2}$ J. Hylen, ${ }^{2}$ V. Papadimitriou, ${ }^{2}$ R. Zwaska, ${ }^{2}$ D. Senor, ${ }^{3}$ \\ A. Casella, ${ }^{3}$ and D. J. Edwards ${ }^{3}$ \\ ${ }^{1}$ Brookhaven National Laboratory (BNL), Upton, New York 11973, USA \\ ${ }^{2}$ Fermi National Accelerator Laboratory, Batavia, Illinois 60510, USA \\ ${ }^{3}$ Pacific Northwest National Laboratory, Richland, Washington 99354, USA \\ ${ }^{4}$ Stony Brook University, Stony Brook, New York 11794, USA \\ ${ }^{5}$ National Center for Scientific Research “Demokritos," Athens, Greece
}

(Received 22 August 2018; published 8 April 2019)

\begin{abstract}
The NT-02 neutrino physics target made of the isotropic graphite grade produced neutrinos for the MINOS and MINERVA high-energy physics experiments. The segmented, 95-cm-long NT-02 target was bombarded with a $340 \mathrm{~kW}$, Gaussian $1.1 \mathrm{~mm}$ sigma beam of $120 \mathrm{GeV}$ protons reaching $6.516 \times 10^{20}$ protons on target and a peak fluence of $8.6 \times 10^{21}$ protons $/ \mathrm{cm}^{2}$. Reductions in detected neutrino events during the experiment were attributed to radiation-induced damage on the target material leading to the NT-02 target replacement. With future neutrino physics targets aiming at the multimegawatt power regime, identifying life expectancy or fluence thresholds of target materials is of paramount importance, and, therefore, pinpointing the exact cause and target failure mode triggering the neutrino yield reduction is critical. To help unravel the effects of the $120 \mathrm{GeV}$ beam on the isotropic graphite structure at the microstructural or lattice level, $\mathrm{x}$-ray beams from National Synchrotron Light Source II were utilized to study failed in-beam as well as intact NT-02 target segments. The primary objective was to arrive at a scientifically sound explanation of the processes responsible for the target failure by correlating macroscopic observations with microstructural analyses. Results from transmission electron microscopy studies were integrated in assessing the microstructural evolution. The x-ray diffraction study revealed (a) the diffused state reached by the graphite microstructure within the $1 \sigma$ of the beam where the graphite lattice structure transforms into a nanocrystalline structure, a finding supported by electron microscopy examination, thus providing an indication of the fluence threshold, and (b) the dominant role of the irradiation temperature profile exhibiting a high gradient from the beam center to the heat sink and aggravating the damage induced in the microstructure by the high proton fluence. The effects of the $120 \mathrm{GeV}$ protons on the isotropic graphite target structure are corroborated by observed damage induced by $160-\mathrm{MeV}$ protons and by fast neutrons to comparative doses on similar graphite, an assessment that will aid the design of next-generation megawatt-class neutrino targets.
\end{abstract}

DOI: 10.1103/PhysRevAccelBeams.22.041001

\section{INTRODUCTION}

The NT-02 neutrino physics target in the NuMI beam line at Fermilab (Fig. 1) produced neutrinos for the MINOS and MINERVA high-energy physics experiments. The shape of the $340 \mathrm{~kW}, 120 \mathrm{GeV}$ proton beam delivered on target was Gaussian with a $1.1 \mathrm{~mm} \sigma$. The 95-cm-long target (length chosen for two beam interaction lengths) was

Published by the American Physical Society under the terms of the Creative Commons Attribution 4.0 International license. Further distribution of this work must maintain attribution to the author(s) and the published article's title, journal citation, and DOI. made of the isotropic graphite grade POCO ZXF-5Q and was segmented into 47 fins $(6.6 \mathrm{~mm} \times 15 \mathrm{~mm} \times 20 \mathrm{~mm})$ along the proton beam path, with each fin brazed at the top and bottom on a stainless-steel pipe providing circulating water as the heat sink. With the $10 \mu$ s beam pulse length and a cycle time of $1.87 \mathrm{~s}$, the graphite target was estimated, based on proton transport and subsequently thermal analyses, to experience rapid temperature cycling from $\sim 60^{\circ} \mathrm{C}$ to $\sim 370^{\circ} \mathrm{C}$. The NT-02 target operated within a noncirculating helium environment. During the exposure of the NT-02 to the $120 \mathrm{GeV}$ beam, $6.516 \times 10^{20}$ protons were delivered on target resulting in a peak fluence of $\sim 8.6 \times 10^{21}$ protons $/ \mathrm{cm}^{2}$.

A reduction in the detected neutrino yield during the experiment was detected. Specifically, over the latter half of 


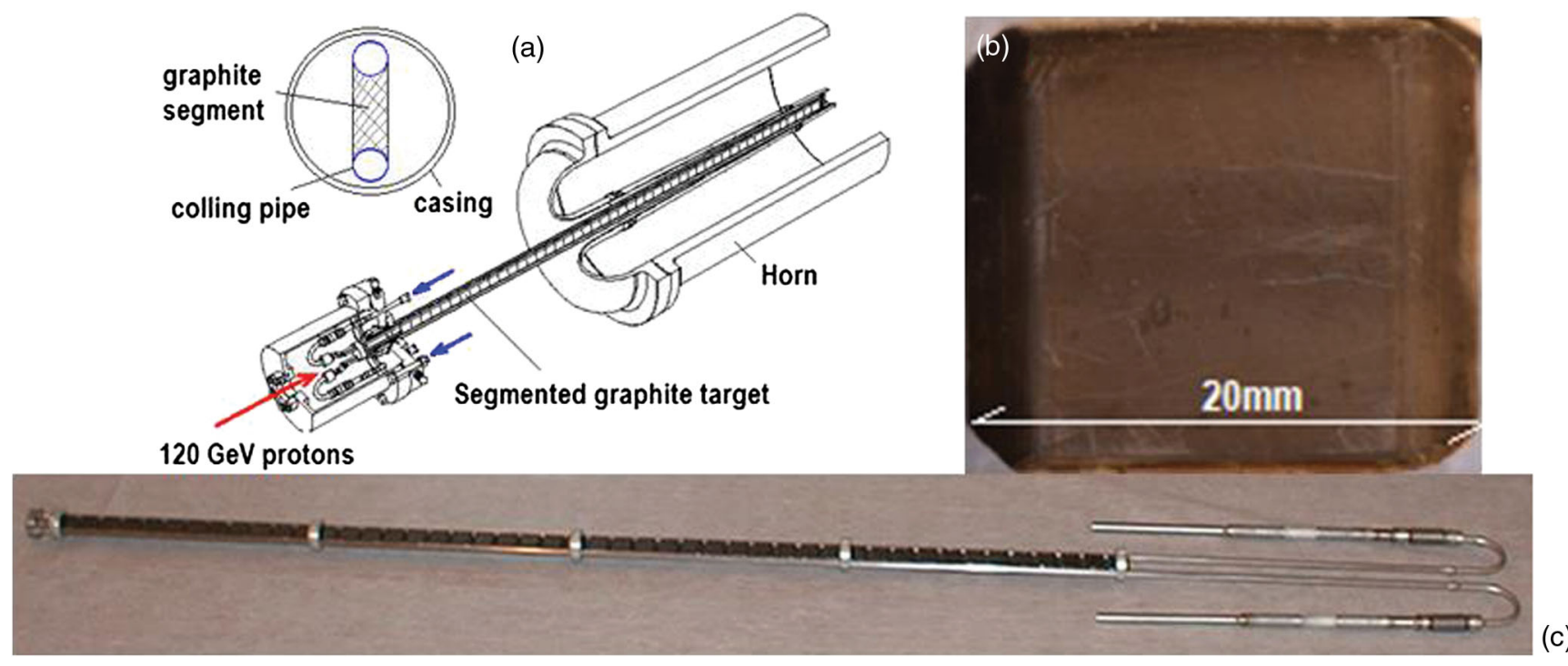

FIG. 1. (a) Neutrino (NuMI) target configuration, (b) intact NT-02 target fin following irradiation to $8.6 \times 10^{21} \mathrm{p} / \mathrm{cm}^{2}$, and (c) photoimage of the actual NT-02 target depicting the full array of fins.

the NT-02 target's lifetime, a gradual decline in the neutrino yield of $\sim 10 \%-15 \%$ was observed, prompting its eventual replacement. This reduction was attributed to radiationinduced damage in the graphite microstructure within the thin cylinder traversed by the $1.1 \mathrm{~mm} \sigma$ beam that progressively led to macrocracking and consequently a density reduction, which, in turn, affected the optimal spectrum of liberated pions and kaons captured by the magnetic field surrounding the target. Upon target retrieval and examination and inspection, global cracks shown in Fig. 2(b) were evident in several upstream fins (upstream target edges with the first three fins in the sequence are shown). The fracture surface along a failed in-beam target fin is depicted in Fig. 2(a). It is estimated that the shown fracture surface in Fig. 2(a) is that of fin 2 in the sequence. A postmortem evaluation of the NT-02 target revealed bulk swelling of the order of $\sim 2 \%$ (relative swelling between the center and fin edges) to have taken place in fins located both upstream and downstream with more swelling associated with upstream locations and fractured fins. The examination confirmed that target fin fractures have occurred during the $120 \mathrm{GeV}$ beam irradiation.

Evidently, severe in-beam radiation damage in the $95-\mathrm{cm}-$ long target could have potentially pushed target segments located in the downstream region close to fracture or failure limits. This stems from the fact that fins retrieved from the downstream half also failed either in beam or during subsequent retrieval or detachment from the stainless-steel cooling pipes they were brazed onto for examination.

A large body of research has emerged over the past seven decades regarding graphite and the effects of thermal and fast neutrons on its micro- and macrostructure as well as its thermophysical properties [1-19]. Changes in physical and mechanical properties, swelling, and annealing as well as evolution of the graphite lattice structure were addressed. Dimensional changes, swelling, and mechanical properties were reported in Refs. [1-5]. The activation energy for annealing is reported in Ref. [6]. Studies on the lattice parameter evolution under neutron and/or electron irradiation were reported in Refs. [7-16]. Nuclear graphite

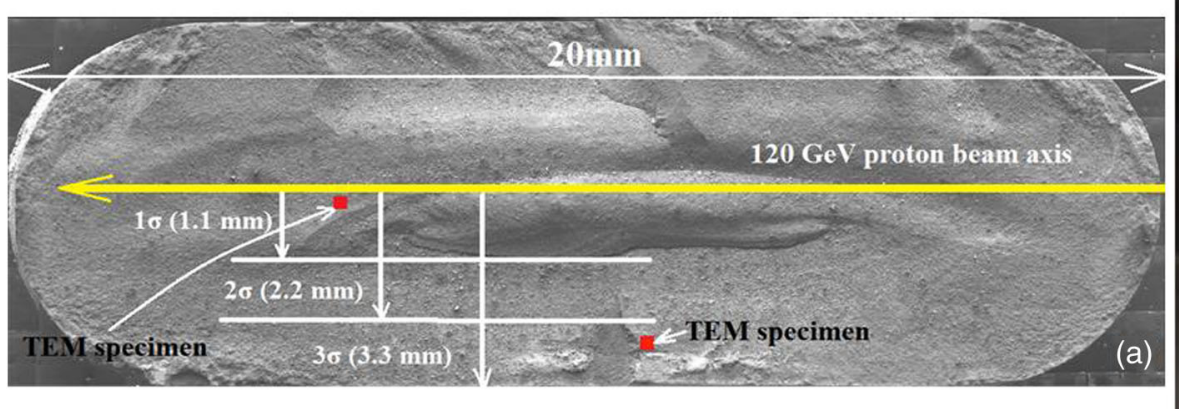

FIG. 2. NuMI target NT-02 upstream (a) fin fracture surface and (b) macrocracking fin failure.

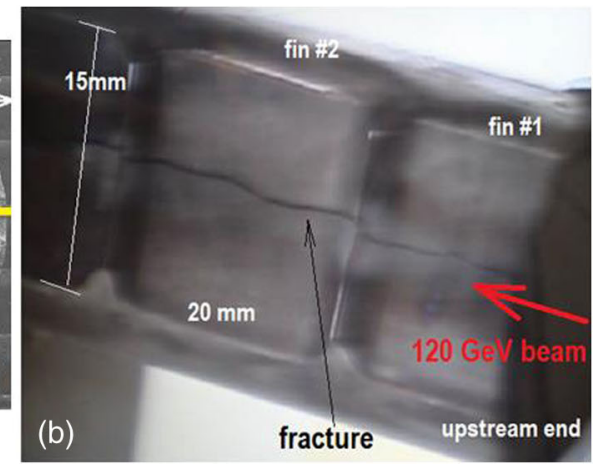


irradiated at high temperatures was studied in Ref. [17]. Creep and swelling of nuclear graphite under neutron irradiation were studied in Ref. [18]. The effects of heavy ion bombardment on graphite and its effect on the lattice structure are presented in Ref. [19]. It is this wealth of operational and experimental evidence from neutron-based irradiation of graphite which achieved fluences that exceed even the NT-02 peak proton fluence that the authors are tapping into to help explain the proton irradiation-induced damage of the NuMI target.

Several studies [20-24] focusing on energetic proton irradiation effects on the physiomechanical properties and the changes in the microstructure have been conducted in recent years. This is prompted by the fact that graphite, in its various grades including POCO ZXF-5Q of the NuMI target, has been used in several neutrino experiment initiatives (NuMI/MINOS, T2K, and CNGS) as a low-Z material producing a desired pion spectrum. Because of its low $Z$, good resistance to thermal shock, and track record in surviving high neutron fluences in fission reactors, graphite has been a primary candidate for use in several high-power accelerator initiatives. Because of its lattice structure, however, graphite undergoes changes in important physical properties under irradiation at relatively low doses and low temperatures, and, thus, this regime of dose temperature warrants special attention.

Specifically, and prior to the experience of the NuMI graphite target in a beam which led to a high proton fluence $\left(\sim 8.6 \times 10^{21} \mathrm{p} / \mathrm{cm}^{2}\right)$, neutron-based experimental studies have succeeded over several decades in reaching fluence levels $\left(>10^{22} \mathrm{n} / \mathrm{cm}^{2}\right)$ that were impossible to achieve using accelerator protons due to the prohibitive cost. Consequently, a "correlation" of the damage induced by the irradiating species (neutrons and protons) has been sought, research that continues to be of great interest to date, to facilitate the extrapolation of proton effects on graphite and other materials using the experience from surrogate species, such as neutrons at extreme fluences. In recent studies [22], lattice parameter and physical property changes have been directly compared for similar fluence and irradiation temperatures of fast neutrons and $160-\mathrm{MeV}$ protons. It was concluded [22] that irradiation effects on the graphite are equivalent. In the present study, however, where the proton energy is $120 \mathrm{GeV}$ (i.e., several orders of magnitude higher than the energy of fast neutrons), the use of an "unverified" extrapolation or correlation between the irradiating species is not prudent. In other words, the direct use of graphite damage observed from a few $\mathrm{MeV}$ neutrons to similar fluence $\left(\sim 10^{22} \mathrm{n} / \mathrm{cm}^{2}\right)$ to assess damage and/or microstructural evolution in the NuMI graphite target exposed to $120 \mathrm{GeV}$ protons may lead to wrong assessments as to the cause of target failure. To bridge this gap, postirradiation, postmortem microstructure-focused experiments such as x-ray diffraction and electron microscopy were necessary.
To that end, high-resolution x-ray diffraction (XRD) of high-energy $\mathrm{x}$ rays $(67 \mathrm{keV})$ was conducted at the X-ray Powder Diffraction (XPD) beam line of National Synchrotron Light Source (NSLS) II, the world's brightest synchrotron light source. XRD experiments were performed on failed in-beam and intact fins of the $120 \mathrm{GeV}$ NT-02 target. These $\mathrm{x}$-ray-based experiments were augmented with transmission electron microscopy (TEM) studies conducted at Pacific Northwest National Laboratory (PNNL) on TEM samples extracted from the proton-affected zone. The primary objective of both studies was to interpret the findings on the effects the $120 \mathrm{GeV}$ proton beam had on the microstructure into physiomechanical changes in the graphite and pinpoint the failure driving mechanism. Irradiation damage and microstructural evolution induced by $160-\mathrm{MeV}$ protons on the identical isotropic graphite grade (POCO ZXF-5Q) were also utilized as guidance in assessing the effects under $120 \mathrm{GeV}$ protons.

Section II summarizes relevant results of past studies [21-24] on the POCO ZXF-5Q and other isotropic graphites from high neutron and proton fluences. We shall use these data as the basis for interpreting the changes induced by the $120 \mathrm{GeV}$ proton beams. This, in turn, can help assess the NT-02 target failure. In Sec. III, the results of the comprehensive experimental $\mathrm{x}$-ray study on the NT-02 intact and failed-in-beam target fins are presented. Section IV presents the integration of x-ray diffraction results with transmission electron microscopy observations coupled with predictions of the beam-induced state of the NuMI target based on transport codes towards establishing the most probable target failure scenario. Finally, in Sec. V, assessment and conclusive remarks regarding a high-power target design based on the NuMI target performance in beam, the design parameters that are considered to be dominant, and the limitations of the material choice based on the irradiation damage studies to date are presented.

\section{CHARACTERIZATION OF NT-02 TARGET POCO ZXF-5Q GRAPHITE}

In this section, the irradiation temperature and its spatial variation or gradient over the segmented, 95-cm-long NT02 target are estimated to establish a basis for (a) thermophysical characterization of the POCO ZXF-5Q graphite structure over the temperature regime of interest, (b) correlation with $160-\mathrm{MeV}$ proton damage of the exact same grade graphite to doses lower than the $120 \mathrm{GeV}$ peak dose but equivalent to doses outside the $1 \sigma$ zone, and (c) correlation and relevance with neutron-based experimental data and damage on a similar (isotropic) graphite structure from fluences in excess of $8.6 \times 10^{21} \mathrm{p} / \mathrm{cm}^{2}$ and for different irradiation temperatures.

The temperature profile in the target during irradiation was assumed to be a major contributor to the response and eventual failure of the target and, more importantly, the steep temperature gradient between the center of the 
$1.1 \mathrm{~mm} \sigma$ proton beam and the heat sink (cooled edge). Energy deposition estimates deduced using the transport codes FLUKA [25,26] and MARS [27] produced similar results while providing a detailed spatial distribution of deposited energy throughout the 95-cm NT-02 target. Figure 3(a) depicts the energy deposition profile at midplane of the target. Figure 3(b) shows the evolution of the peak energy density and the total energy deposited in each of the segments. The profile of the energy deposition on a plane normal to the proton beam is depicted in Fig. 3(c) at several locations. The energy profile shown is indicative of the temperature variation between the beam center and the heat sink that exists at either end (top and bottom) of each segment fin. It is evident that a very steep variation of the temperature between the center of the proton beam and the region outside the $2 \sigma$ exists, particularly in the upstream section of the target. Progressively, the differences ease within the downstream half of the target. Specifically, it is estimated that for $4.0 \times 10^{13}$ protons per spill during NuMI operation (spill $=10 \mu \mathrm{s}$ ) the peak energy deposited in the target downstream of the first fin is $\sim 480 \mathrm{~J} / \mathrm{cm}^{3}$ per spill ( $\sim 265 \mathrm{~J} / \mathrm{g}$ per spill). A three-dimensional finite element transient analysis of the thermomechanical response of a target fin resulting from the rapid energy deposition by the $10-\mu \mathrm{s}$ beam spill was performed to assess both the temperature transients and resulting stresses in the graphite target fin. The capabilities of the LS-DYNA explicit dynamics software [28] were utilized for the analyses based on a high-fidelity finite element model. Energy deposition in the studied NT-02 target segment (peak energy density location was selected per Fig. 3) was introduced into the finite element model of the target segment that was assumed to be constrained at the top and bottom (cooling water supply pipes) and with appropriate temperature boundary conditions at the two ends (those of coolant supply and return temperatures). The transient thermomechanical analysis revealed the following: The peak temperature rise within the $1 \sigma$ of the proton beam is estimated to be $\sim 384^{\circ} \mathrm{C}$. The estimated thermal stresses developing from each arriving beam pulse reach $\sim 31 \mathrm{MPa}$ in compression (maximum dynamic) and $\sim 14 \mathrm{MPa}$ in tension. These maxima stresses are experienced by each target fin near the midsection, where cracks were observed. The peak tensile stresses were shown to occur near the edges of each fin.

To assess whether fatigue in the target fin had played a dominant role in the failure (cracking that was observed), the transient stresses calculated from the analysis were considered along with the number of stress cycles
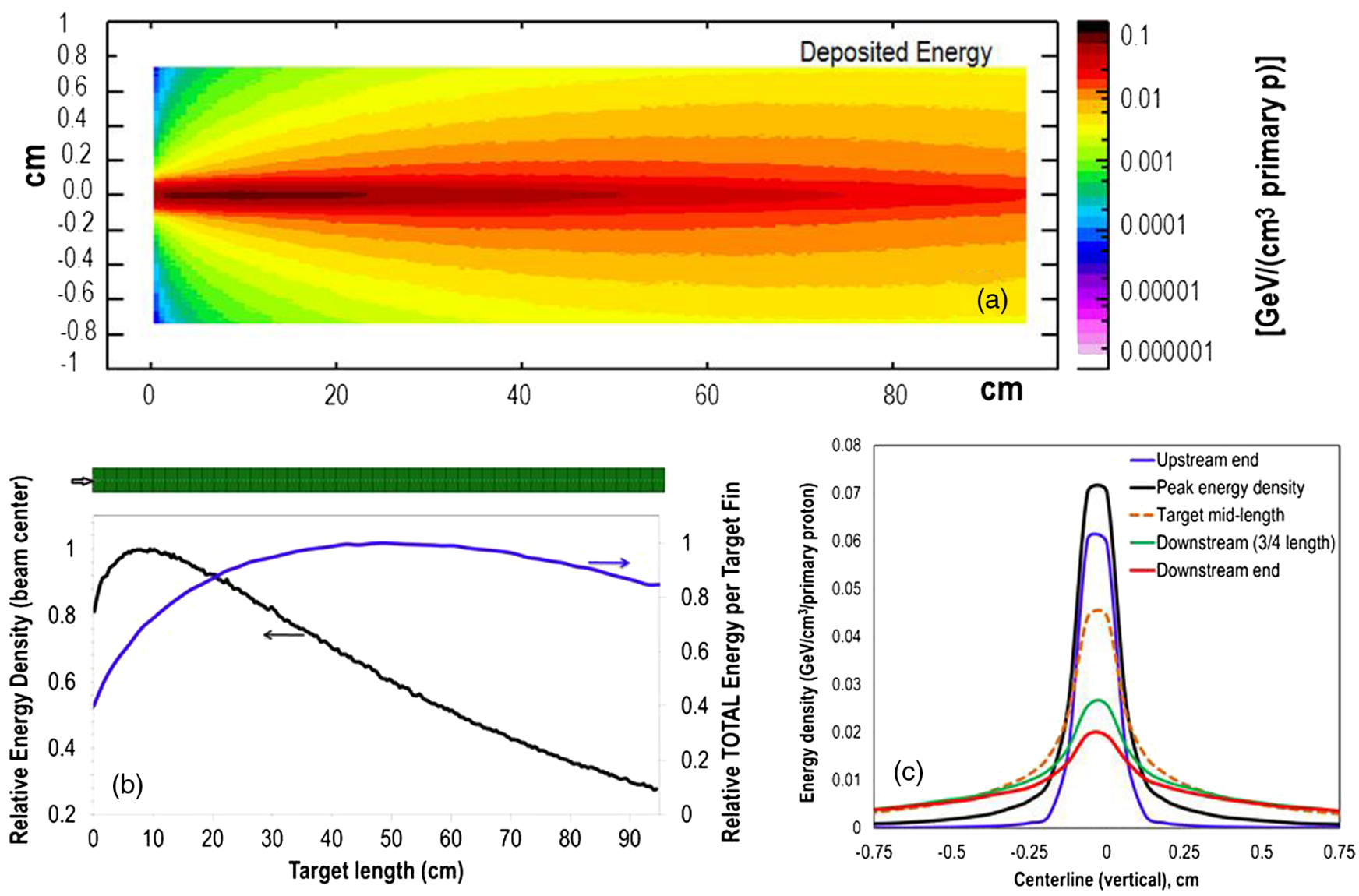

FIG. 3. (a) NuMI target NT-02 energy deposition profile from the $120 \mathrm{GeV}$ proton beam in midplane, (b) relative energy density and energy deposited, and (c) energy deposition profile normal to the beam. 
experienced by the target during its operation. Based on the integrated time in a beam of the NT-02 target and the cycle time of $1.87 \mathrm{~s}$, that estimated number of pulses is $\sim 1.4 \times 10^{7}$. The compressive strength of the unirradiated target graphite material is $\sim 170 \mathrm{MPa}$, but the tensile strength is much lower, $75 \mathrm{MPa}$. Both strength limits are expected to initially increase with irradiation before they experience a reduction above a fluence threshold $[12,22]$. Even though the cyclic stress estimated to be experienced by the target fins appears to be small, as compared to the maxima in strength, it is the authors' opinion that fatigue may still be considered as a contributing factor to the overall response and failure of the target fins, just based on the excessively high stress cycles experienced. This assessment can help explain the fracture of fins located in the downstream half, whether they had already fractured in the beam or were close to fracture from fatigue-induced strength reduction and failed due to handling during disassembly. An experimental effort has been launched to obtain fracture surface strengths via nanoindentation and which, along with numerically obtained stress evolution, provides vital information on the role or contribution of fatigue.

\section{A. Thermal analysis and scanning electron microscopy characterization}

Scanning electron microscopy (SEM) was conducted on POCO ZXF-5Q and two other graphites (IG-430 and AC-150) (Fig. 4) to compare populations of Mrozowski cracks and their evolution with an increasing temperature. It has been established in several studies $[1,3,6,8,11]$ that, during low fluence irradiation, densification driven by the fact that growth in the $c$ direction results in filling preexisting Mrozowski cracks is taking place. Macroscopic shrinkage is observed, because vacancies in the basal plane result in $a$-parameter shrinkage. Swelling will begin after the Mrozowski cracks are filled and the $c$-parameter increase starts to dominate. Three graphitic structures, shown in Fig. 4, have been closely examined via scanning electron microscopy to qualitatively assess their microstructure. Close examination of the micrographs reveals that significantly fewer Mrozowski cracks are present in the unirradiated POCO ZXF-5Q graphite as compared to IG430 and the graphite matrix of the compound Mo-GR, implying that swelling, rather than shrinkage, will dominate in the POCO ZXF-5Q from the onset of irradiation.

Additionally, thermal analyses differential scanning calorimetry and thermogravimetric analysis (TGA) were conducted in an argon atmosphere on the POCO ZXF-5Q grade to observe its thermal response and weight loss in the temperature regime between $\sim 60^{\circ} \mathrm{C}$ and $370{ }^{\circ} \mathrm{C}$, where the NuMI target NT-02 operated. As seen in Fig. 5, depicting the TGA analysis results of heat flow and weight change for the temperature range up to $1000{ }^{\circ} \mathrm{C}$, no dramatic changes are seen to occur within that temperature regime to have any contribution towards the target failure.

\section{B. Irradiation damage from neutrons and $160-\mathrm{MeV}$ protons}

Irradiation-induced swelling resulting from the bombardment of graphite by neutrons has been a subject of extensive research [1-7] driven by the interest in the behavior of nuclear graphite. Macroscopic swelling and cell parameter changes reveal that the irradiation temperature has a profound effect on both macroscopic and lattice level changes. Depicted in Fig. 6(a) [4] are dimensional changes in isotropic graphite irradiated with fast neutrons at $150^{\circ}$ C. Shown in Fig. 5(b) [15] are macroscopic dimensional changes in isotropic graphite for several irradiation temperatures. Similar to what has been established by several studies [1-3,6-8,13-16] at the lattice level as a result of the stored energy release that essentially begins at $\sim 200^{\circ} \mathrm{C}$, the two distinct temperature regimes (below $200^{\circ} \mathrm{C}$ and above $200^{\circ} \mathrm{C}$ ) are also observed for the macroscopic behavior of isotropic graphite [Fig. 5(b)]. At irradiation temperatures below $200^{\circ} \mathrm{C}$, the isotropic graphite tends to swell rather than shrink, a process typically observed in other nonisotropic grades prompted by the filling, initially, of the pores and Mrozowski cracks. At irradiation temperatures above $300^{\circ} \mathrm{C}$, the isotropic-type graphite [Fig. 6(b)] experiences macroscopic shrinking for all doses. POCO ZXF-5Q, a highly isotropic grade, is expected to also adhere to the trend.
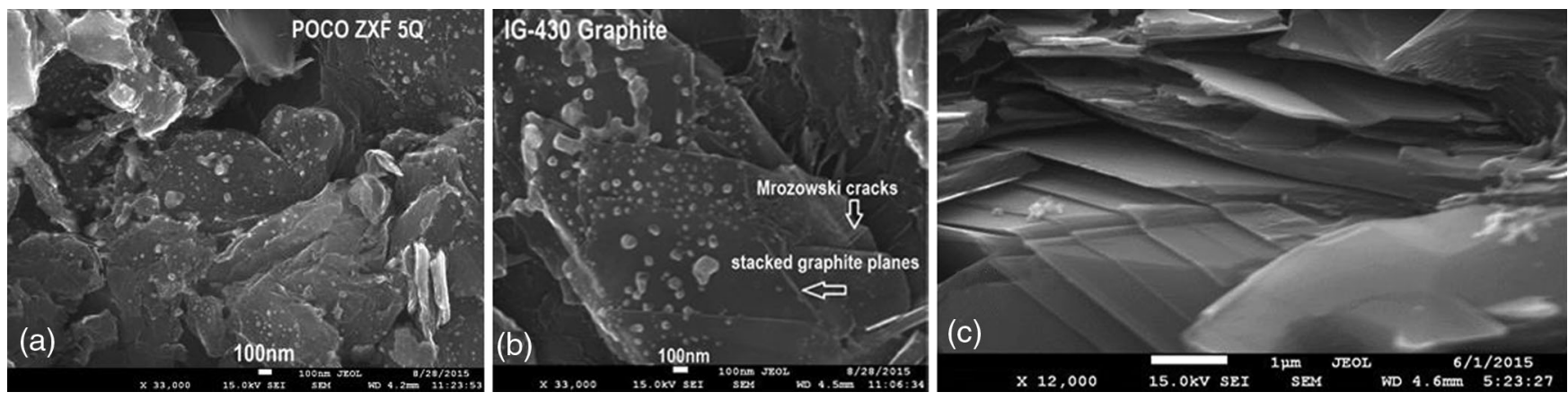

FIG. 4. SEM micrographs of (a) POCO ZXF-5Q, (b) IG-430, and (c) graphite matrix in Mo-graphite compound. 

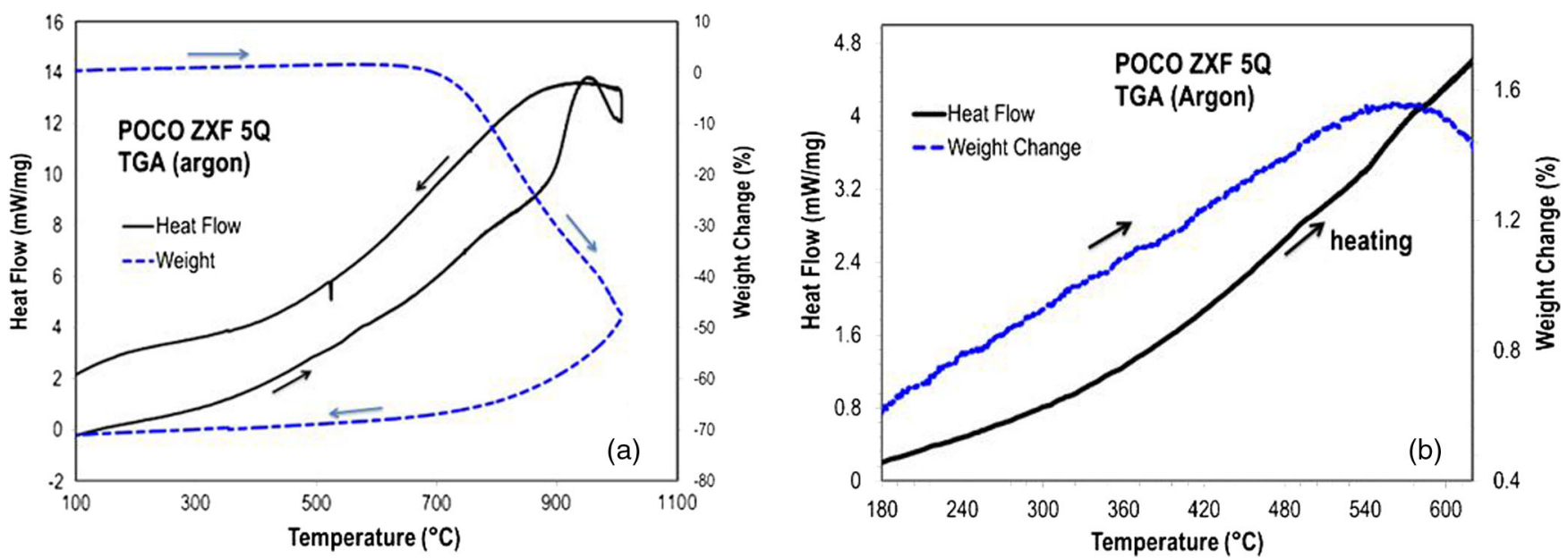

FIG. 5. Thermal analysis (TGA) of POCO ZXF-5Q in an argon atmosphere. (a) Complete thermal cycle to $1000{ }^{\circ} \mathrm{C}$ and (b) close-up view of the $180-600^{\circ} \mathrm{C}$ range (note range of scale of weight change percent).
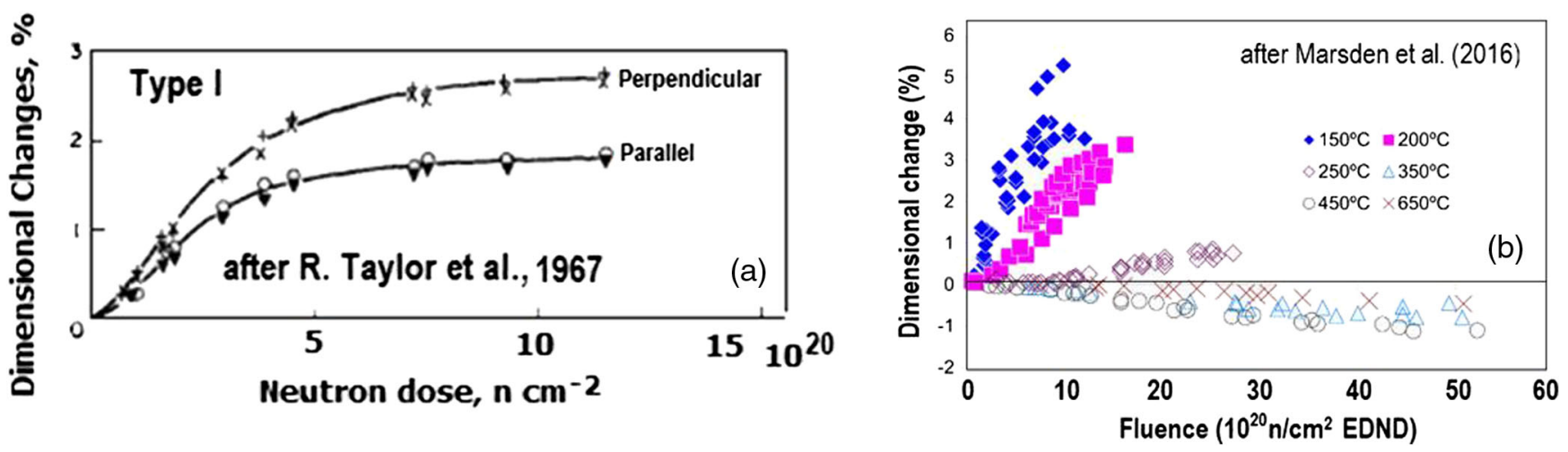

FIG. 6. Macroscopic dimensional changes in isotropic graphite grades irradiated with fast neutrons at various irradiation temperatures (after Refs. [4,14]).

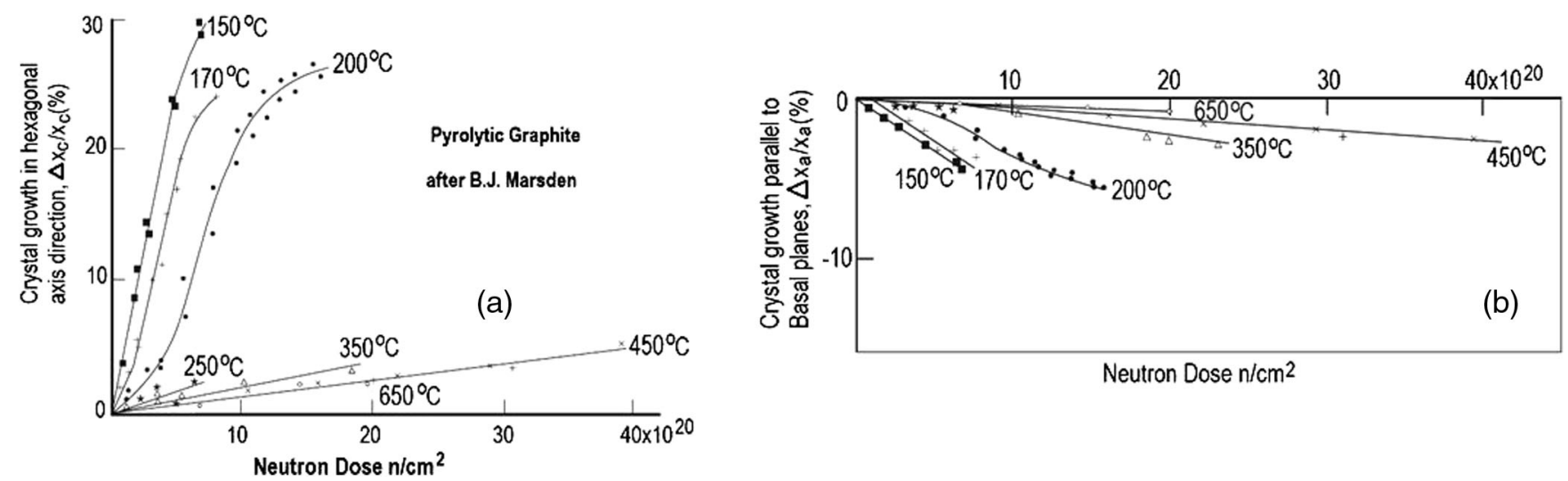

FIG. 7. Lattice changes in the (a) $c$ axis and (b) $a$ axis of isotropic graphite by fast neutrons [13].

Lattice parameter changes in pyrolytic graphite [14] for neutron fluences up to $\sim 40 \times 10^{20} \mathrm{n} / \mathrm{cm}^{2}$ and a wide range of irradiation temperatures are depicted in Fig. 7 [11]. As seen in Fig. 7(a), the $c$ axis at temperatures at and below $200^{\circ} \mathrm{C}$ increases dramatically even for low neutron doses.
At temperatures above the $200{ }^{\circ} \mathrm{C}$ threshold, the increase is significantly reduced and appears to be linear with the dose at a given irradiation temperature. The $a$-axis parameter undergoes shrinkage [Fig. 7(b)] but at a smaller rate while showing the two distinct temperature regions. 

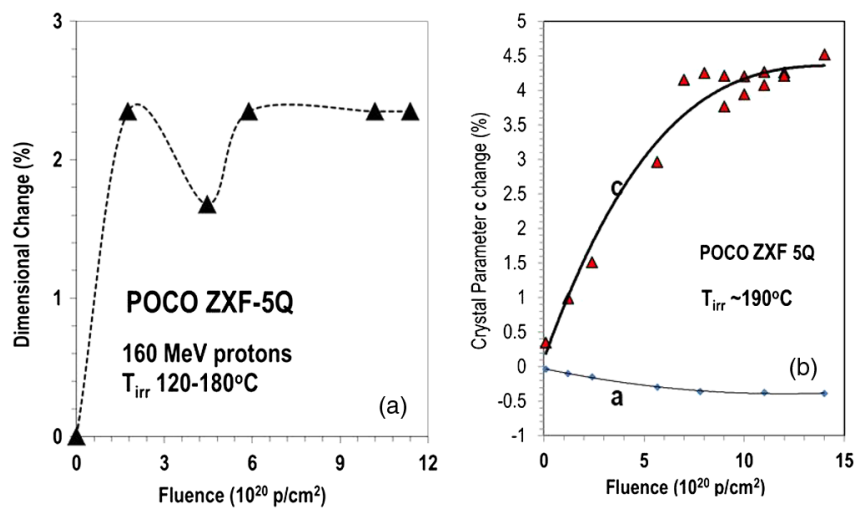

FIG. 8. (a) Swelling of POCO ZXF-5Q graphite irradiated with $160-\mathrm{MeV}$ protons and (b) the lattice parameter change.

To ensure that the isotropic POCO ZXF-5Q of the NuMI target adheres to trends depicted in Figs. 6 and 7 regarding macroscopic and microscopic dimensional changes, irradiation damage experiments were conducted at BNL using $160-\mathrm{MeV}$ protons on this graphite grade and compared with those reported $[4,13,14]$. Specifically, during the 160$\mathrm{MeV}$ experiment, $\sim 3.2 \times 10^{21}$ protons were delivered on target translating to a peak fluence of $\sim 1.1 \times 10^{21} \mathrm{p} / \mathrm{cm}^{2}$ with irradiation temperatures estimated (both numerically and during postirradiation annealing) in the $120-$ $200( \pm 20){ }^{\circ} \mathrm{C}$ range in an argon atmosphere. The macroscopic dimensional changes induced in the POCO ZXF-5Q graphite were assessed using a combination of precision micrometers and ultrasonic techniques. Ultrasonic techniques also reveal damage and elastic modulus changes correlated to ultrasonic velocity evolution with damage.

Figure 8(a) depicts dimensional changes as a function of the fluence of $160-\mathrm{MeV}$ proton-irradiated POCO ZXF-5Q. Similar swelling is observed in agreement with that reported for neutron-irradiated isotropic graphite (Fig. 6). The lattice $c$-parameter change of POCO ZXF-5Q graphite irradiated with $160-\mathrm{MeV}$ protons at $\sim 190{ }^{\circ} \mathrm{C}\left( \pm 20^{\circ} \mathrm{C}\right)$ is shown in Fig. 8(b). These $c$-parameter change results are also in good agreement with changes induced in other isotropic graphites under fast neutron irradiation. The corresponding changes in the $a$ parameter of POCO ZXF-5Q due to $160-\mathrm{MeV}$ protons are also shown in Fig. 7(b) $\left(\Delta a=-0.38 \%\right.$ for $\left.\sim 1.14 \times 10^{21} \mathrm{p} / \mathrm{cm}^{2}\right)$, deviating from those from pyrolytic carbon under similar neutron irradiation [Fig. 7(b)] in terms of the amplitude of the change. Such a deviation in the amplitude of the $a$-parameter change may stem from the difference in graphite type (pyrolytic vs $\mathrm{POCO}$ ), the irradiating species (fast neutrons vs protons), and, most importantly, the sensitivity of the lattice parameter changes with a small variation of the irradiation temperature around the $200{ }^{\circ} \mathrm{C}$ threshold. Assigned temperatures in the proton-irradiated results are average temperatures over the $6 \mathrm{~mm}$ gauge of the macroscopic specimen (estimated), while the x-ray beam diffraction was performed at the central part of the gauge that coincides with the peak of the proton beam Gaussian shape, implying that the actual temperature over the volume being interrogated was potentially higher than the estimated average. As Fig. 7 clearly demonstrates, small variations in the irradiation temperature around the $200{ }^{\circ} \mathrm{C}$ threshold are accompanied by large differences in $a$-parameter and $c$-parameter changes as a function of the fluence.

The supporting experimental studies reported thus far in this section confirm that POCO ZXF-5Q isotropic graphite follows the trends in terms of macroscopic swelling, lattice changes observed in studies to date following neutron and proton irradiation. The most important confirmation of its behavior under irradiation is the dimensional changes as a function of the irradiation temperature for isotropic graphite. The dramatic differences in swelling between the lowtemperature regime $\left(<200^{\circ} \mathrm{C}\right)$ even at low irradiation and the high temperature $\left(>200^{\circ} \mathrm{C}\right)$ for high irradiation doses are directly relevant to the NT- 02 target. This is expected to hold the key in explaining the observed failure of NT-02 and the variation in behavior even when the temperatures are considered small by other standards.

\section{IRRADIATED NT-02 TARGET X-RAY DIFFRACTION EXPERIMENT}

Two NT-02 target fins are studied using the $67 \mathrm{keV} x$ rays at the NSLS II XPD beam line. One fin, located at the upstream (US) position in the target, experienced in-beam fracture. The other fin is a whole and intact segment located at the downstream (DS) target section. The two specimens are shown in Fig. 9 along with the x-ray scanning scheme that was performed at the XPD beam line.

The $67 \mathrm{keV}$ x-ray beam with a spot of $0.5 \mathrm{~mm} \times 0.5 \mathrm{~mm}$ can traverse the entire length of the fin $(20 \mathrm{~mm})$ with minimal diffusion. Furthermore, the x-ray beam is aligned along the $z$ direction with the trace of the proton beam through the fin. Precise diffraction measurements with $500 \mu \mathrm{m}$ resolution at different distances away from the proton beam center were made in both the vertical $(Y)$ and the horizontal $(X)$ directions of the fin cross section (as shown in Fig. 9) with identical exposures to the X-ray beam. A precisely controlled stage at the XPD (28 ID-2) beam line enabled the scanning of the photon beam in the vertical $(Y)$ direction between the two cooled edges of the target fin and through the proton beam center.

This important experimental feature allowed for a precise comparison of the microstructure between the upper and lower halves of the intact DS fin to help establish the effect of possible temperature profile variation stemming from the inlet-outlet cooling temperature differences at the two cooled edges of the intact fin. Deviations from a "centered" beam, in both the vertical and horizontal axes, are established on the basis of the shape of the diffraction patterns. The $\mathrm{x}$-ray analysis is expected to also reveal differences between the US fractured half fin and its DS counterpart due to anticipated differences in the proton fluence and, 


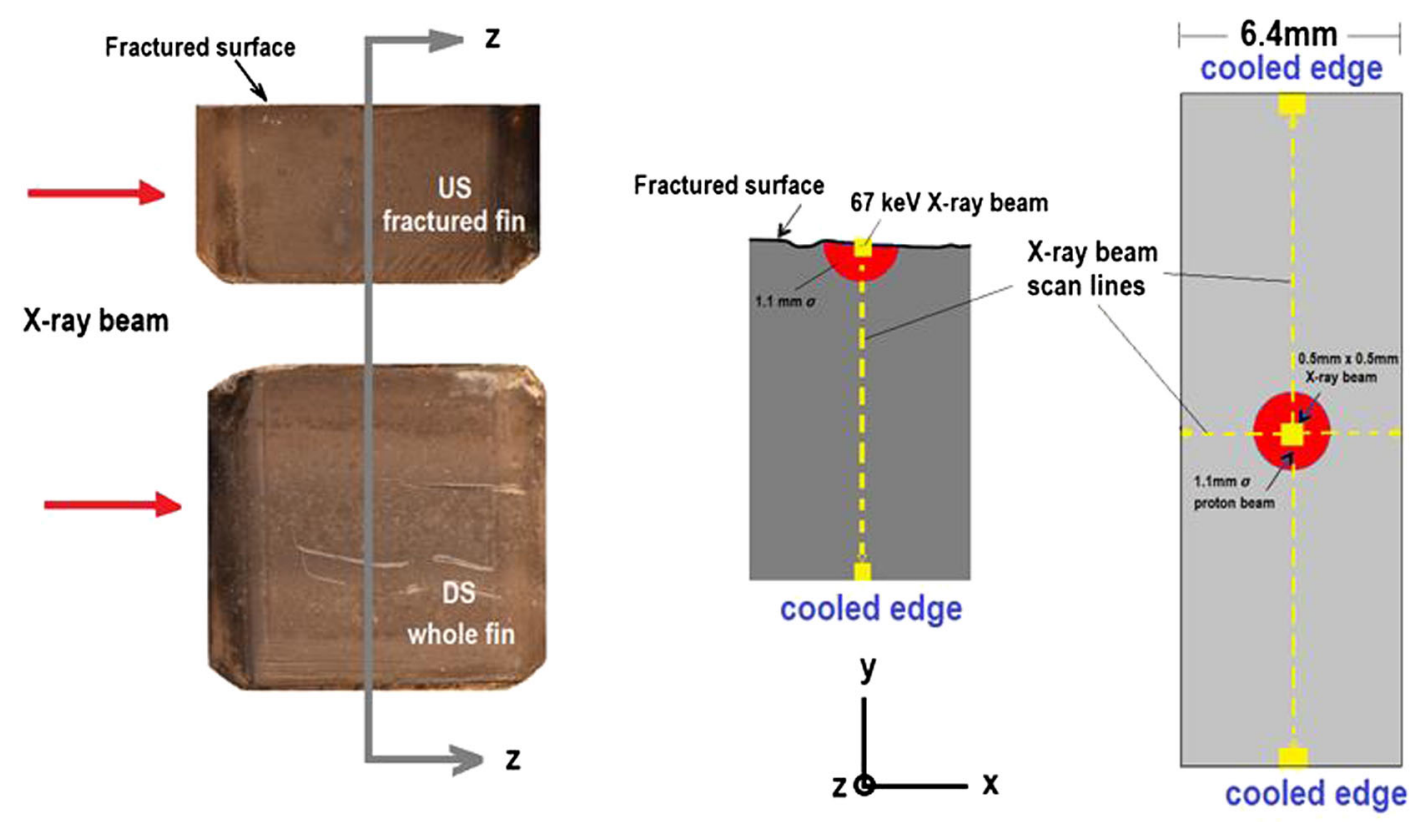

FIG. 9. NT-02 fin sample x-ray scanning scheme.

consequently, differences in the irradiation temperature magnitude and profile [Fig. 3(c)]. Proton transport analyses $[26,27]$ within the two interaction lengths of the $120 \mathrm{GeV}$ beam with the $95-\mathrm{cm}$-long graphite target indicated a significant reduction $(>50 \%)$ in the proton fluence towards the downstream section of the target [see Fig. 18(a)].

Selected x-ray diffraction images through the NT-02 specimens are depicted in Figs. 10 and 11. Figure 10(a)
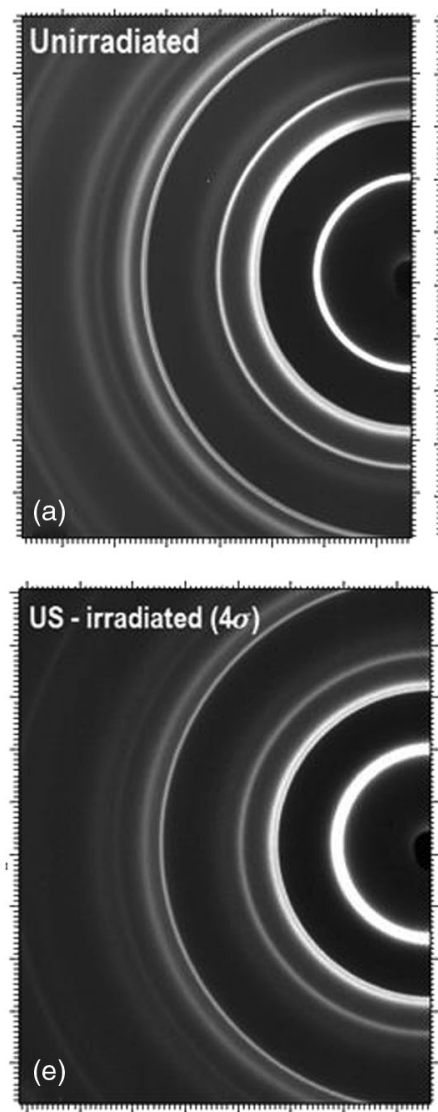
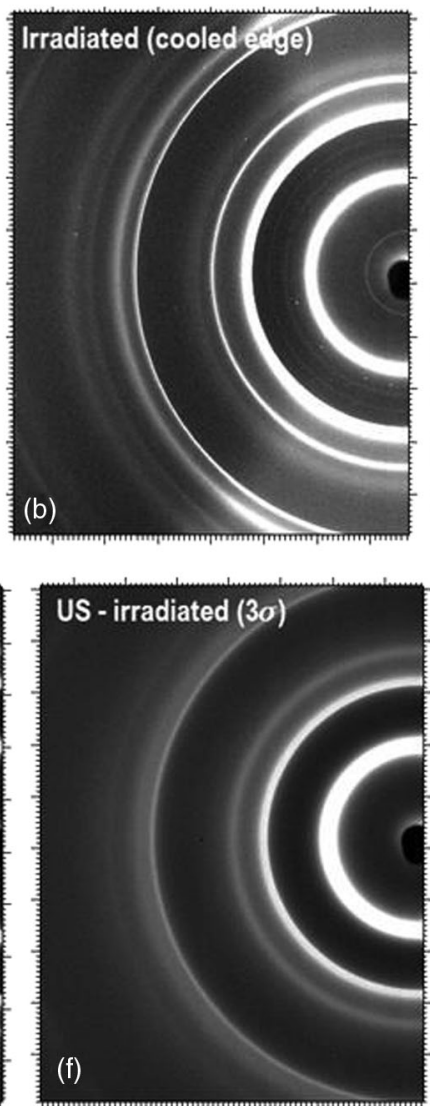
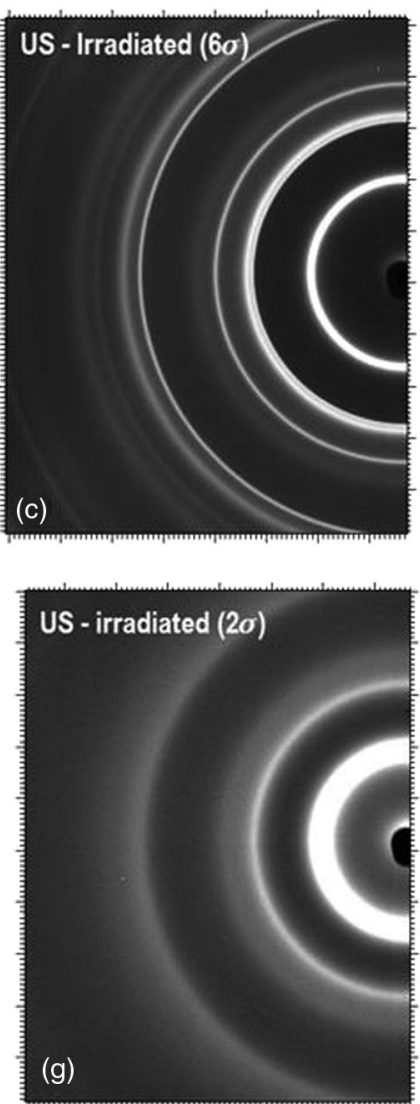
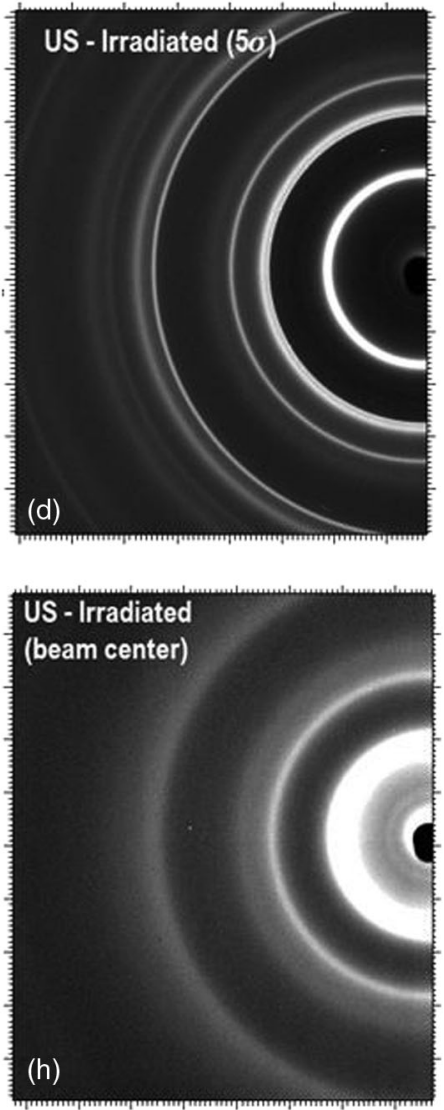

FIG. 10. Phase evolution relative to the $120 \mathrm{GeV}$ proton beam in the US target failed in beam. 

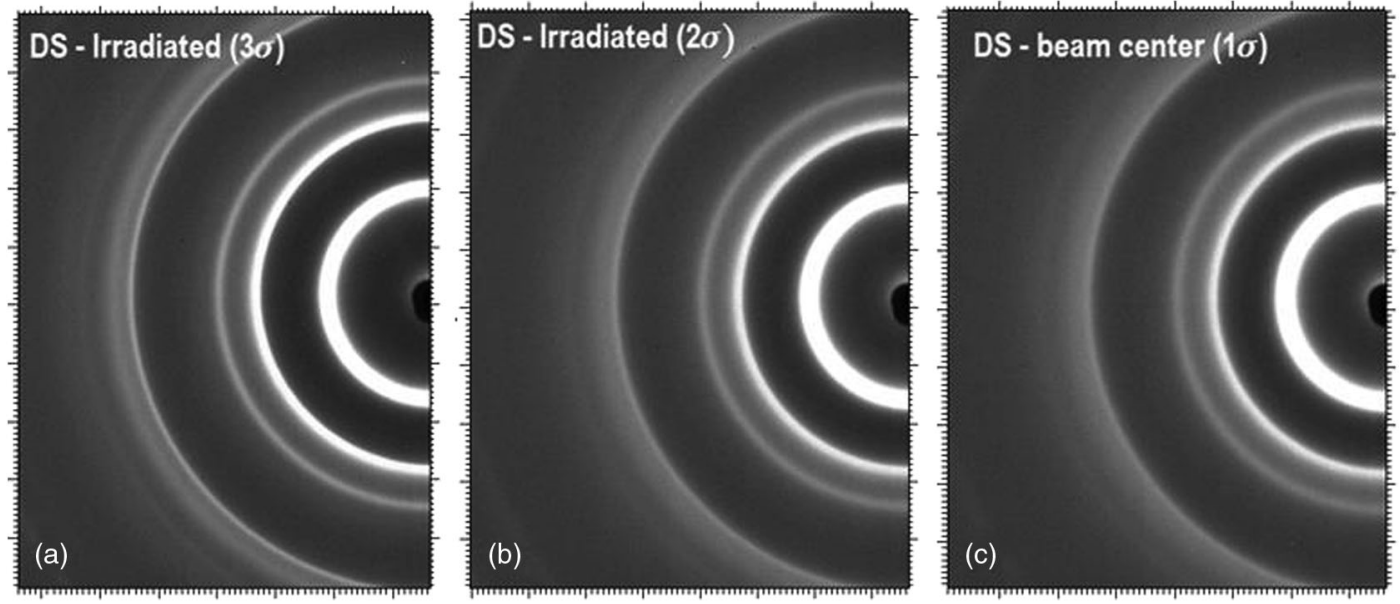

FIG. 11. Phase evolution in the intact DS target segment at (a) $3 \sigma$, (b) $2 \sigma$, and (c) the beam center.

depicts the 2D detector image of unirradiated POCO ZXF5Q graphite sample serving as the baseline. Figure 10(b) depicts the 2D diffraction image at the cooled edge of the US fractured target fin. Diffraction image evolution from $6 \sigma$ to the beam center at the fracture surface is shown in Figs. 10(c)-10(h). The series of images in Fig. 10 reveals the pronounced variation of the appearance of the diffraction rings when probing the material from the outer edge (far from the irradiation zone) towards the center (where the proton beam interacted with the target). The changing diffraction rings (in intensity and widths) reflect the increasing loss of crystallinity towards the center of the target and, in particular, the diffused state of the (002), (004), and (210) reflections at the fracture surface.

In contrast, as shown in Fig. 11(c), the diffraction image at the beam center of the DS intact fin has experienced significantly less crystallinity loss than the US counterpart. A comparison of the 2D diffraction images shown in Fig. 11 with those of the US target segment in Fig. 10 reveals that the state of the graphite structure within $1 \sigma$ in the DS (intact) segment is similar to the one observed over the $3 \sigma$ position [Fig. 10(f)] of the failed upstream segment.

The evolution of the US, fractured target fin structure is carefully evaluated by studying the changes in the position, shape, and/or disappearance of, primarily, the (002) reflection representing the graphite basal planes. The 2D diffraction images in Figs. 10 and 11 could be processed and transformed into "conventional" $2 \theta$-intensity 1D diffraction patterns shown, for example, in Figs. 12 and 13 and subsequent figures.

A shift in the (002) reflection peak indicates an increase in the distance between basal planes owing to radiation damage. A decrease in the peak integrated intensity and asymmetric broadening indicate an increasing disordering, loss of crystallinity, and lattice distortion and strain. Finally, the absence of the (002) and (004) reflection reveals that the graphite structure turned amorphous and without longrange order and that the graphite crystals have fragmented into small, disordered grains. Figures 12 and 13(a) offer clear evidence that the US target fin has essentially amorphized over most of the cross section. The crystalline structure, as seen in Fig. 13(b), is also lost within the basal plane. We may suspect that the crystalline-to-amorphous structure transformation at the exact location where the material was hit by the proton beam was a factor in the rupturing of this part of the target. One other important finding is the "turnaround" in the (002) $d$ spacing (or parameter $c$ ) approaching the cooled edge. This confirms the important effect of the irradiation temperature in addition to the dose dependence.

Shown in Fig. 14(a) is the evolution of the (002) reflection over the entire height along the $Y$ direction

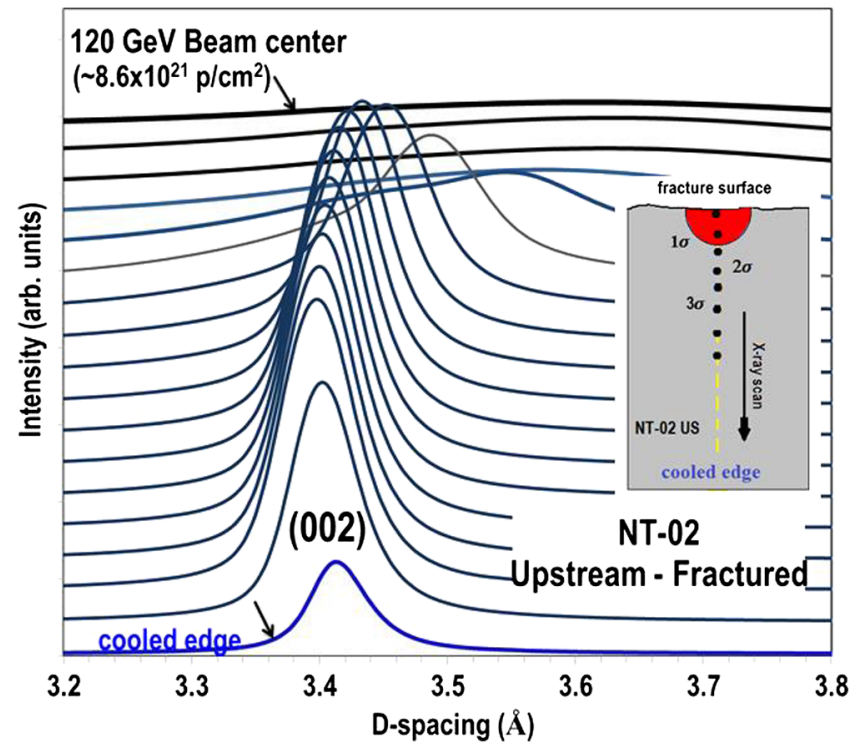

FIG. 12. Evolution of the (002) crystallographic plane of the top half of the US target fin from the proton beam center (peak fluence $8.6 \times 10^{21} \mathrm{p} / \mathrm{cm}^{2}$ ) to the cooled edge (inset, scanning scheme). 

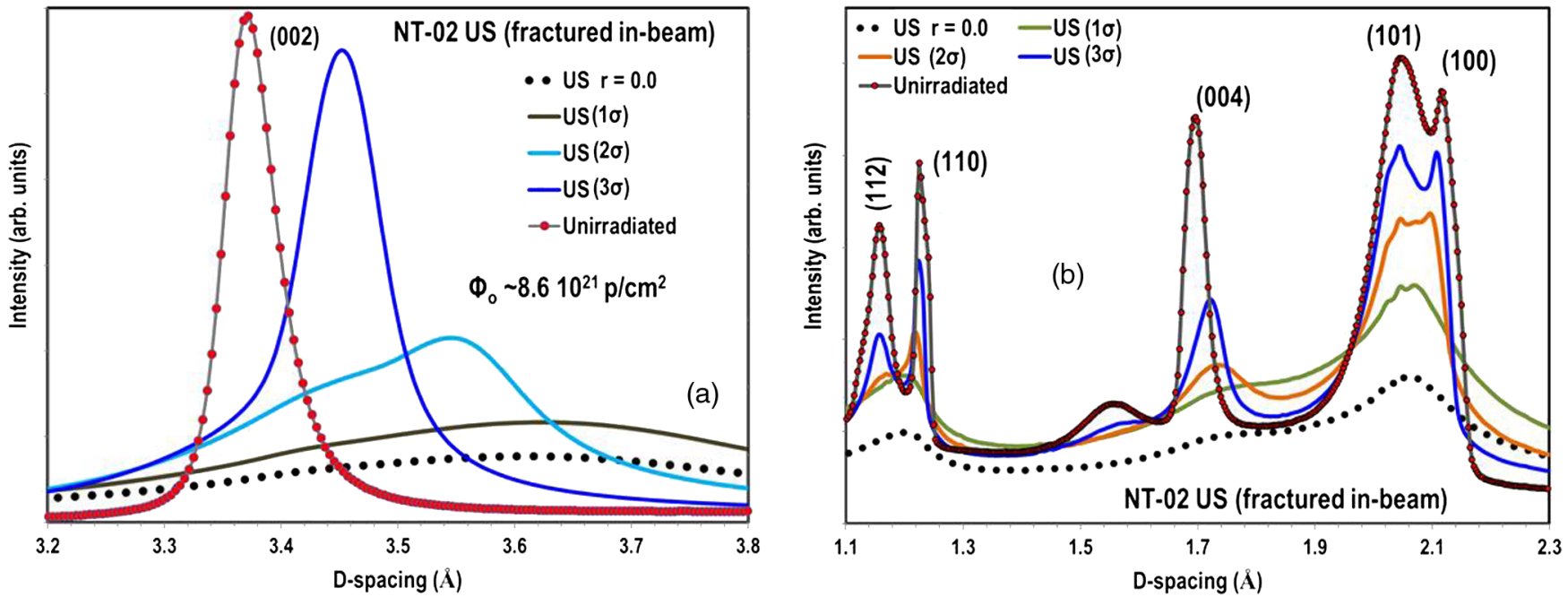

FIG. 13. (a) Evolution of crystallographic (002) plane at locations from the proton beam center to $3 \sigma$ distance of the US, failed target fin, and comparison with the unirradiated state, and (b) evolution of several plane reflections and comparison with the unirradiated state.
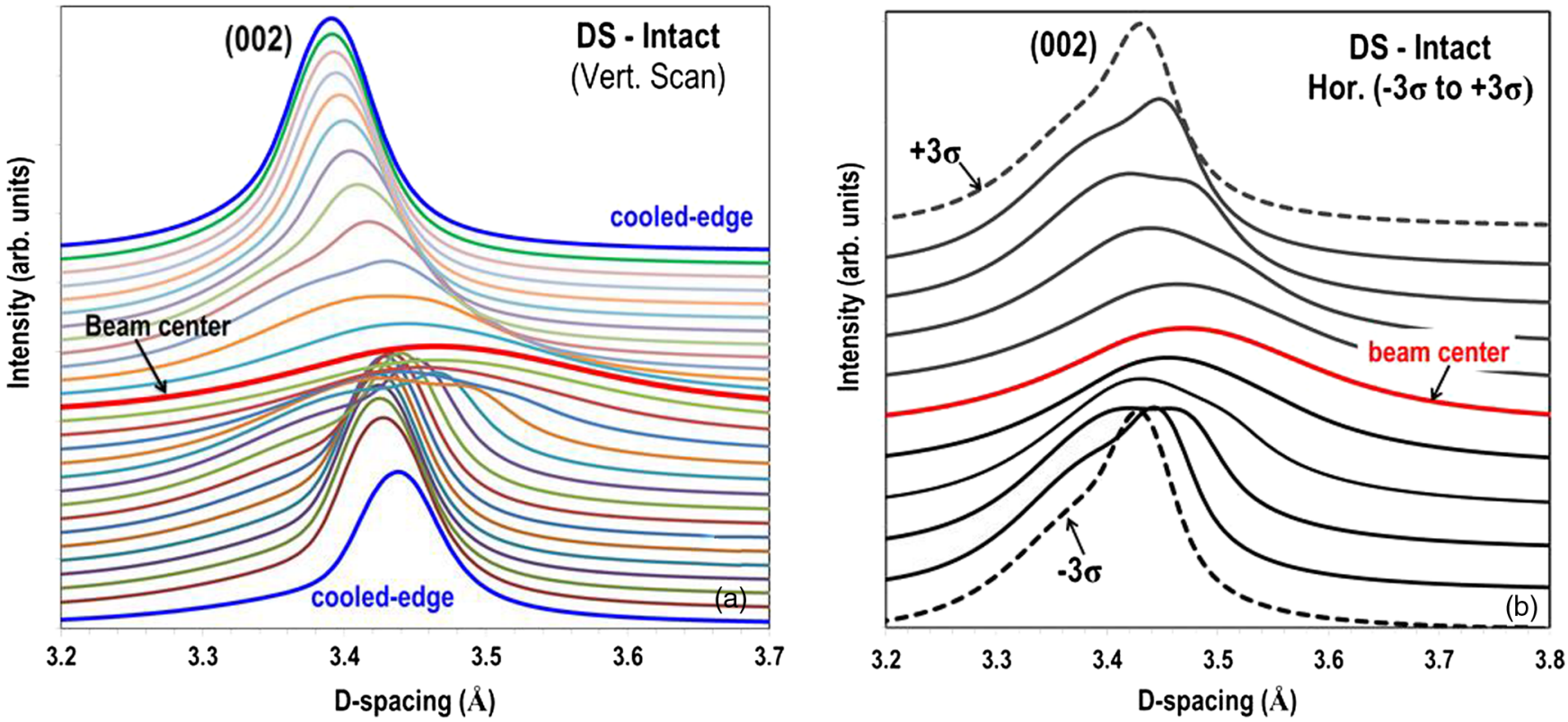

FIG. 14. (a) Evolution of the (002) crystallographic plane along the vertical $Y$ axis of the downstream (DS, intact) target fin and (b) along the horizontal ( $X$ axis) at midheight of the intact fin $(-3 \sigma$ to $+3 \sigma)$.

(top cooled edge to bottom cooled edge) of the downstream (DS, intact) fin and in Fig. 14(b) the evolution along the horizontal ( $X$ axis) at midhigh. Figure 14(a) clearly reveals peak asymmetric broadening and peak position shifting. The top half and the evolution trend is remarkably similar to the US fractured fin shown in Fig. 12. In both cases, a turnaround in the (002) reflection is detected near the cooled edge. Monitoring of the cooling water flow (heat sink) during the experiment, however, revealed only a modest temperature increase between the input and return of the water loop $\left(\sim 10^{\circ} \mathrm{C}\right)$. Although such $\Delta T$ in the cooling loop is not directly translated into the graphite target fin cooled edge irradiation temperature, it can be utilized as a factor to explain the asymmetry in diffraction observed in Fig. 14(a) between the top and bottom fin halves as well as the turnaround feature. As depicted in Figs. 6(b) and 7, only small variations in temperature may induce disproportionate changes in the graphite structure.

In assessing the mechanism behind the failure, it was important to identify the position of the proton beam relative to the geometric center of the target cross section and to assess the possible beam offset during NuMI operation. Any offset could have a significant implication in the mechanical response of the target aiding the target 

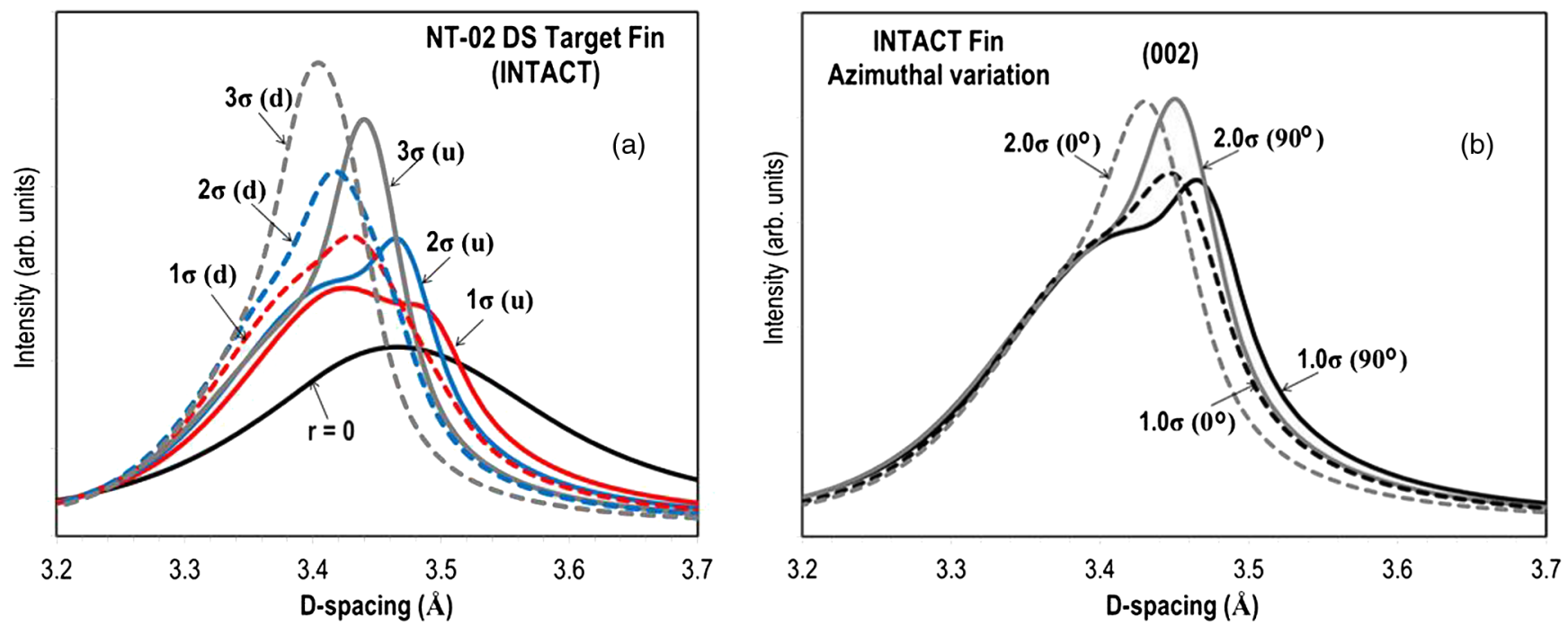

FIG. 15. (a) Observed asymmetry in the (002) reflections between the upper $(u)$ and lower $(d)$ halves of the downstream (DS, intact) target fin and (b) variation of (002) between $0^{\circ}\left(X\right.$ axis) and $90^{\circ}(Y$ axis $)$.

fin failure. While the asymmetric nature of the basal plane (002) reflection is amply evident in Fig. 15(a) along the $Y$ axis (vertical), Fig. 15(b) that depicts the (002) reflection along the horizontal ( $X$ axis) confirms that the proton beam was traversing the target perfectly centered with the horizontal. Figure 15(a) compares (002) reflections at equidistant locations from the center along the $Y$ axis to $3 \sigma$ distance from the target center $(\sigma=1.1 \mathrm{~mm})$ exhibiting significant variation and asymmetry. Figure $15(\mathrm{~b})$, on the other hand, compares the shape of the (002) reflection between $0^{\circ}$ and $90^{\circ}$ positions $\left(90^{\circ}\right.$, the location on the upper half of the DS target fin) at 1 and $2 \sigma$ radii. Figure 15(b) provides strong evidence that the proton beam was indeed centered and round.
In Fig. 16(a), a closer comparison of 1D diffraction patterns (002) between US and DS target fins along with unirradiated and irradiated with $160 \mathrm{MeV}$ to lower fluence POCO ZXF-5Q graphite is presented. It is evident from Fig. 16(b) that the DS, intact fin maintains its crystalline structure even within $1 \sigma$ of the proton beam. Comparison with the $160-\mathrm{MeV}$ proton irradiation conducted at $\sim 190{ }^{\circ} \mathrm{C}$ in Fig. 16(b) reveals that the $c$ axis of the $160-\mathrm{MeV}$ irradiated graphite has increased more even after a much lower fluence, owed to the lower irradiation temperature $\sim 190^{\circ} \mathrm{C}$. From Fig. 16 (a), we can deduce that the $160-\mathrm{MeV}$ irradiation data are relevant to the state of the graphite in the US fin between 2 and $3 \sigma$ ( $3 \sigma$ representing the surface of the target fin in the $Y$ axis). Therefore, macroscopically
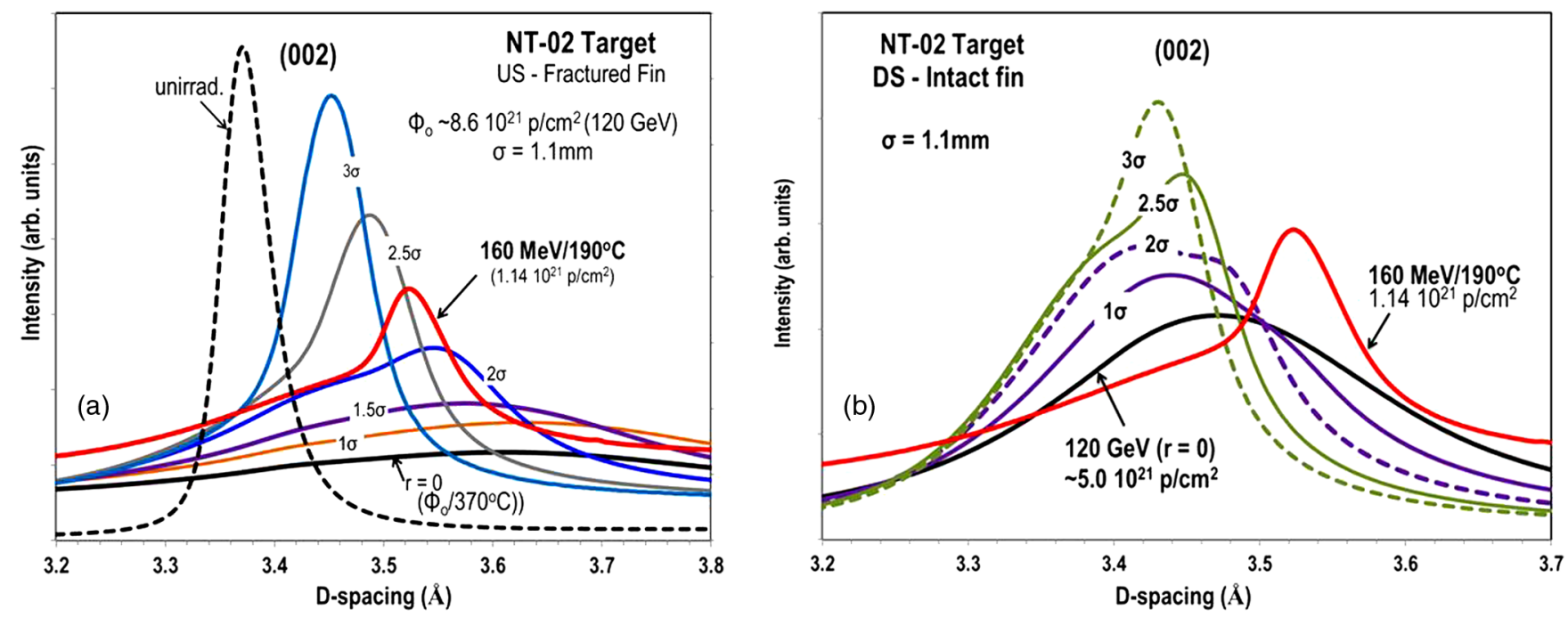

FIG. 16. Comparison of the change in the (002) reflection between locations relative to the $120 \mathrm{GeV}$ beam and that of the same POCO ZXF-5Q grade graphite irradiated with $160-\mathrm{MeV}$ protons at $\sim 190^{\circ} \mathrm{C}$ and to a peak fluence of $1.14 \times 10^{21} \mathrm{p} / \mathrm{cm}^{2}$. 

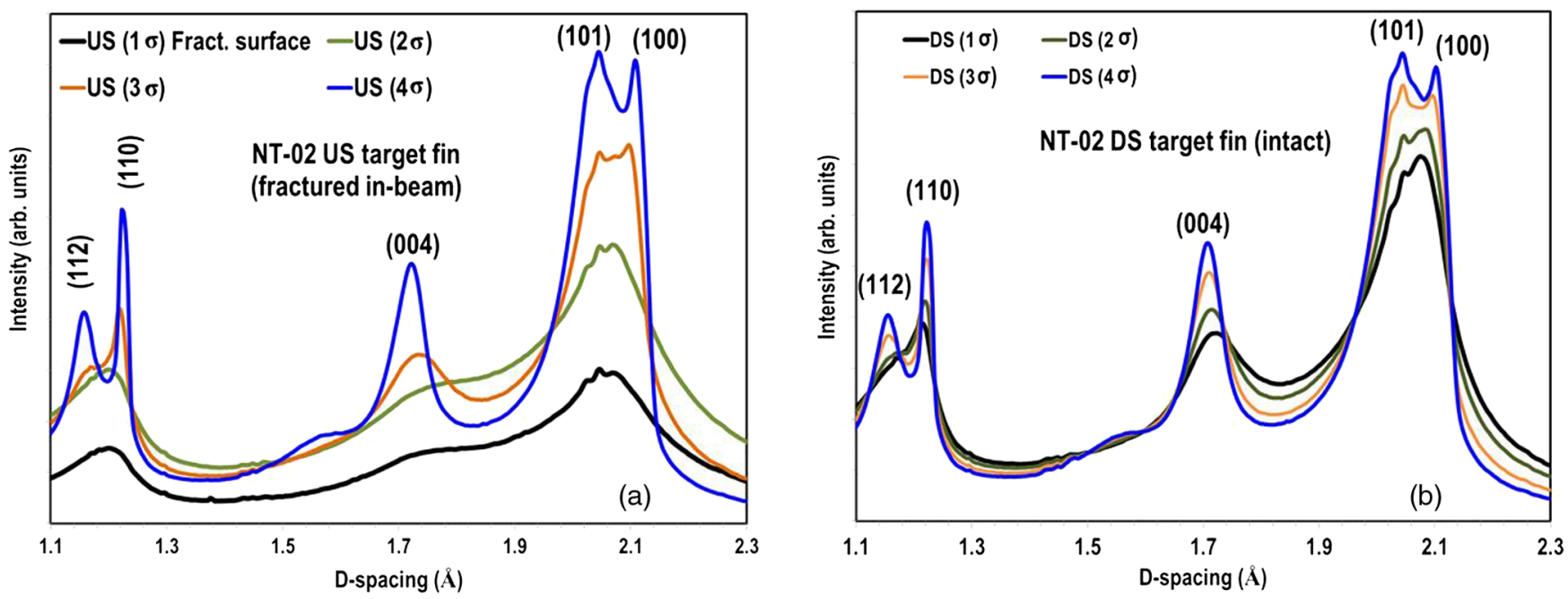

FIG. 17. Comparison of the evolution of several reflections at the same position relative to the proton beam center between the failed upstream target (a) and the intact target (b).

observed changes in the $160-\mathrm{MeV}$ irradiated, same-grade graphite, such as swelling and strength, may be utilized to arrive at the most probable mechanism behind the NT-02 target failure.

As also observed in Fig. 17, which compares the US and DS reflections of planes other than (002), the reflection (110) representing the in-plane atomic distance ( $a$ axis) in the graphite lattice has decreased due to irradiationgenerated vacancies, a process that results in the "shrinking" of the basal plane. The twinning planes (112) have all but disappeared in both US and DS target fins.

\section{NT-02 TARGET FAILURE ASSESSMENT}

Forensic evidence drawn from the NT-02 target fins (failed and intact) consisting of visual inspection, measurements of swelling, and electron microscopy can be summarized as follows:

(i) Target fins in both upstream and downstream sections of the $95 \mathrm{~cm}$ target have been deemed to have failed in-beam.

(ii) The absence or low occurrence of Mrozowski cracks has been observed for this isotropic graphite grade (POCO ZXF-5Q) as compared with other graphites through microscopy (a fact also verified independently in the present study). This is important in that it provides an indication of the tendency to swell or shrink at low-temperature irradiation.

(iii) More swelling was observed in upstream as compared to downstream target fins, a finding possibly related to the reduced fluence in the target downstream.

(iv) More swelling was observed in fractured fins than in their intact counterparts either upstream or downstream, indicating that differential swelling and/or shrinking during irradiation may be the root cause of fracture.
To arrive at an assessment of the NT-02 target failure mode based on the microstructural evolution from the detailed x-ray diffraction experiments of the present study, transmission electron microscopy results from samples extracted from the NT-02 graphite fins and Monte Carlo based transport analysis insights (FLUKA $[25,26]$ and MARS [27]) are integrated into the process. In addition, results from the $160-\mathrm{MeV}$ proton irradiation damage study on the identical grade as well as observations from neutron-based experiments are used. Figure 18 depicts results deduced from the transport code analysis emulating the NuMI target experiment and its interaction with the $120 \mathrm{GeV}$ proton beam. The evolution of proton fluence and the beam profile through the target is shown in Fig. 18(a). It is predicted that the fluence progressively decreases to less than 50\% near the downstream end. Approximately $13 \%$ of protons with kinetic energy within $1 \%$ of the impinging proton energy of $120 \mathrm{GeV}$ are estimated by the transport analysis to leave the target after two interaction lengths [Fig. 2(a)]. These findings are important in comparing the microstructural evolution of the x-ray diffraction tested US (fractured) and DS (intact) fins which were exposed to different fluences and beam profile. Furthermore, the displacement-per-atom (DPA) damage along the target ( $Z$ axis) is shown in Fig. 18(b). Damage $>1$ DPA was estimated to have occurred at the proton beam center in the US section of the target during the NuMI experiment operation. Since comparisons with and use of neutron damage data are made in this study, estimates of spallation neutron damage from the $120 \mathrm{GeV}$ protons are made. Shown in Fig. 18(c) is the fast neutron profile along the NT-02 target indicating that considerable neutron fluence levels are seen by the target fins which are $\sim 8 \sigma$ away from the proton beam center. This may help explain the observed radiation effects at the extremities of the target fins (top and bottom cooled edges). In addition, it should be added that the neutron fluence in 


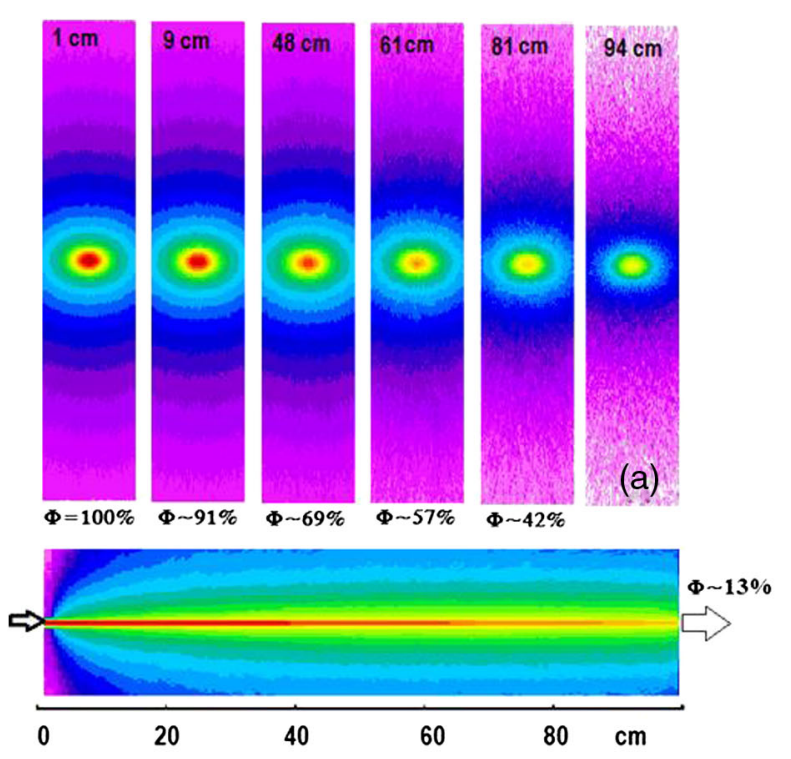

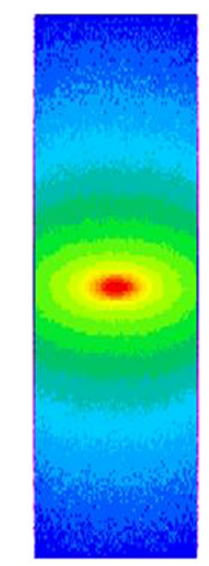

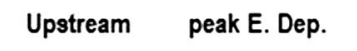

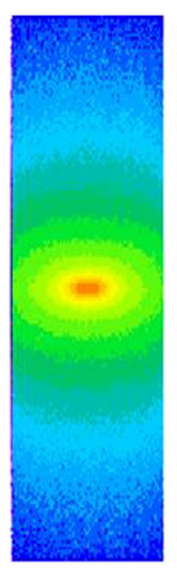

mid-length

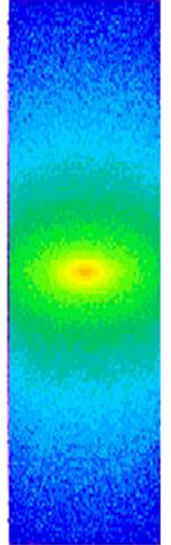

$3 / 4$ length

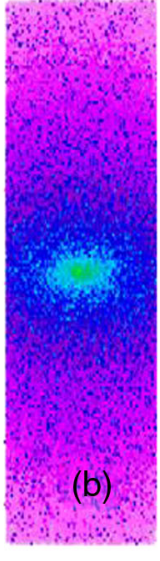

Downstream
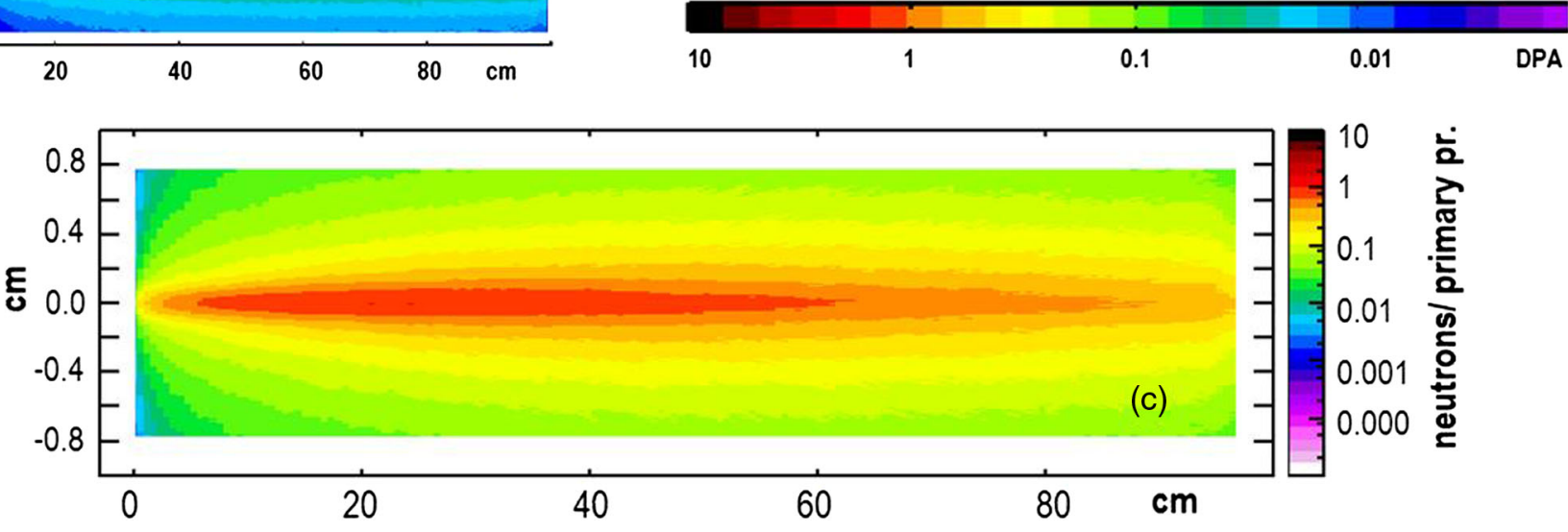

FIG. 18. (a) Prediction of the evolution of the $120 \mathrm{GeV}$ proton beam profile and the degradation of its flux within the two interaction lengths $(95 \mathrm{~cm})$ graphite target including an estimate of primary protons with energy within $1 \%$ of the $120 \mathrm{GeV}$ leaving the target, (b) predicted total displacement damage along the target, and (c) spallation-generated neutron profile in the target.

the upstream part of the 95-cm NT-02 target approaches that of protons, thus effectively pushing the irradiating particle fluence to $\sim 10^{22} \mathrm{~cm}^{-2}$.

\section{A. High-energy x-ray diffraction study}

It is assessed (Figs. 12 and 13) that, due to the absence of the (002) and (004) reflections in the irradiated upstream (DS, failed) target fin, the graphite crystals have fragmented into small, disordered grains and the structure turned amorphous over at least two-thirds of the fin cross section where the proton beam acts. This crystalline-to-amorphous structure transformation has led, we believe, to the excessive weakening of the midsection leading to the observed fracture. The evolution of the strength of the graphite with fluence and of the differential swelling in the target fin, with the latter providing the necessary internally generated forces to induce fracture by pulling the fin apart at midsection, we believe are key factors in the failure mechanism. Therefore, correlation of the evolution under the influence of $120 \mathrm{GeV}$ protons with neutron effects at similar and even higher fluences will provide necessary information on the macroscopic behavior of the graphite in terms of strength and swelling.

Shown in Fig. 19 are the $c$ - and $a$-parameter changes measured for the in-beam fractured fin that experienced the highest fluence $\left(\sim 8.6 \times 10^{21} \mathrm{p} / \mathrm{cm}^{2}\right)$. Figure 19(b) depicts the changes away from the beam center and close to the cooled edge of the US target fin, a section of the fin that sees a small percent of the proton beam at center and is irradiated at much reduced temperatures (estimated $\sim 70{ }^{\circ} \mathrm{C}$ at the cooled end). It should be emphasized that each data point plotted in Fig. 19 corresponds to a distinct fluencetemperature pair stemming from the Gaussian beam shape showering the target. The almost complete amorphization of the graphite crystalline structure in the US fin and at the $120 \mathrm{GeV}$ beam center prevented any $\boldsymbol{c}$ - and $\boldsymbol{a}$-parameter growth estimate at the location. What is evident from Fig. 19(a) is that the growth is not linear with the fluence due to its coupling with the irradiation temperature, an indication that may help explain failure in fins located downstream where the fluence is reduced and irradiation temperatures are lower. Comparing the growth at the $120 \mathrm{GeV}$ proton fluence approaching $\sim 8.0 \times 10^{21} \mathrm{p} / \mathrm{cm}^{2}$ 

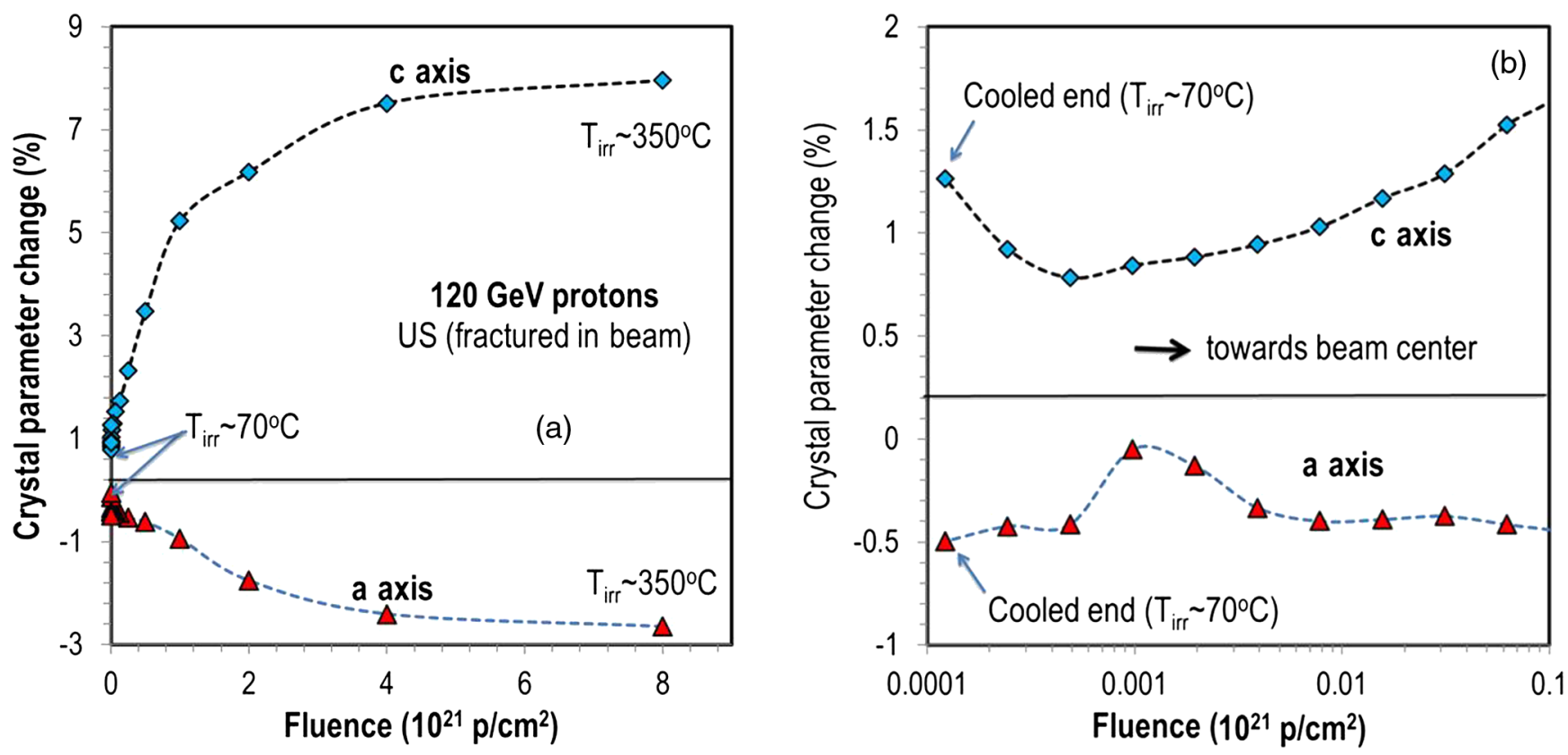

FIG. 19. (a) Graphite crystal parameter changes as a function of the fluence and temperature in the US failed fin and (b) low-fluence region.

and $T_{\text {irr }} \sim 350^{\circ} \mathrm{C}$ with what has been observed for a comparable neutron fluence and irradiation temperature (Fig. 7) for both the $c$ and $a$ axes, it may be concluded that the radiation-induced growth in the crystal is comparable. For the beam center location of the DS target fin, the measured crystal parameter $c$ change is only $\sim 3.3 \%$, which confirms the findings of the transport code analysis regarding the significantly reduced fluence combined with reduced energy deposition resulting in lower irradiation temperatures.

The good agreement between $120 \mathrm{GeV}$ protons and fast neutrons at the graphite microstructure level [Fig. 19(a) $c$ parameter growth compared with the $350{ }^{\circ} \mathrm{C}$ trace in Fig. 7 extrapolated to $8.0 \times 10^{21} \mathrm{n} / \mathrm{cm}^{2}$ ] provides a strong indication that macroscopically the POCO ZXF-5Q isotropic graphite of the NT-02 target adheres to the swelling behavior in Fig. 6(b) [14]. It is, therefore, assessed by the authors that significant internally generated forces develop in the target fin due to differential swelling. Within the beam center and out to a radius of $\sim 2 \sigma$, where temperatures may exceed $300{ }^{\circ} \mathrm{C}$, the isotropic graphite is undergoing shrinkage while adjacent zones at a lower fluence and a lower temperature experience swelling. Swelling has been indeed observed in the fins throughout the target, both upstream and downstream. Target fins subjected to a higher fluence (upstream) are expected to experience more swelling as confirmed by forensic evidence following target failure and retrieval. Also observed is that failed target fins were accompanied by more swelling, which indicates that internally generated forces due to differential swelling were higher. The observation that NT-02 target fins from the downstream half also failed can be explained by the nonlinearity of both the macroscopic swelling or shrinking behavior [Fig. 6(b)] and the crystal lattice changes (Figs. 7 and 19) in terms of the fluence and irradiation temperature. This implies that limiting conditions for the integrity of target fins can arise even in downstream locations where fluences and temperatures are lower.

The evolution of graphite strength with irradiation has also been a subject of study $[3,13,21]$. It has been assessed that graphite strength, under both neutrons and energetic protons, increases with the fluence up to a threshold $\left(\sim 6.0 \times 10^{20} \mathrm{~cm}^{-2}\right)$. Beyond this threshold, graphite strength undergoes a downward trend and at high fluence levels $\left(\sim 10^{22} \mathrm{~cm}^{-2}\right)$ diminishes. This appears to be the case in the US target fin, where complete amorphization of the crystalline structure is observed as the $10^{22} \mathrm{~cm}^{-2}$ fluence is approached, leading to a seriously weakened midsection.

\section{B. Electron Microscopy (TEM)}

Transmission electron microscopy on TEM samples pulled from intact and failed NT-02 target fins were conducted at the PNNL labs to assess the state of the graphite microstructure [26]. A select set of representative micrographs are presented and correlated with the x-ray diffraction results (presented in the previous section) to establish the state of the graphite microstructure (lattice) within the core of the proton beam and away from it. Specifically, the correlation is focused on the failed inbeam target segment for which TEM samples were pulled from the fracture surface at the beam center (within one $\sigma$ ) and at $\sim 3 \sigma$ as shown in Fig. 2. Depicted in Fig. 20(a) is the TEM micrograph at $\sim 3 \sigma$ from the beam center, revealing 

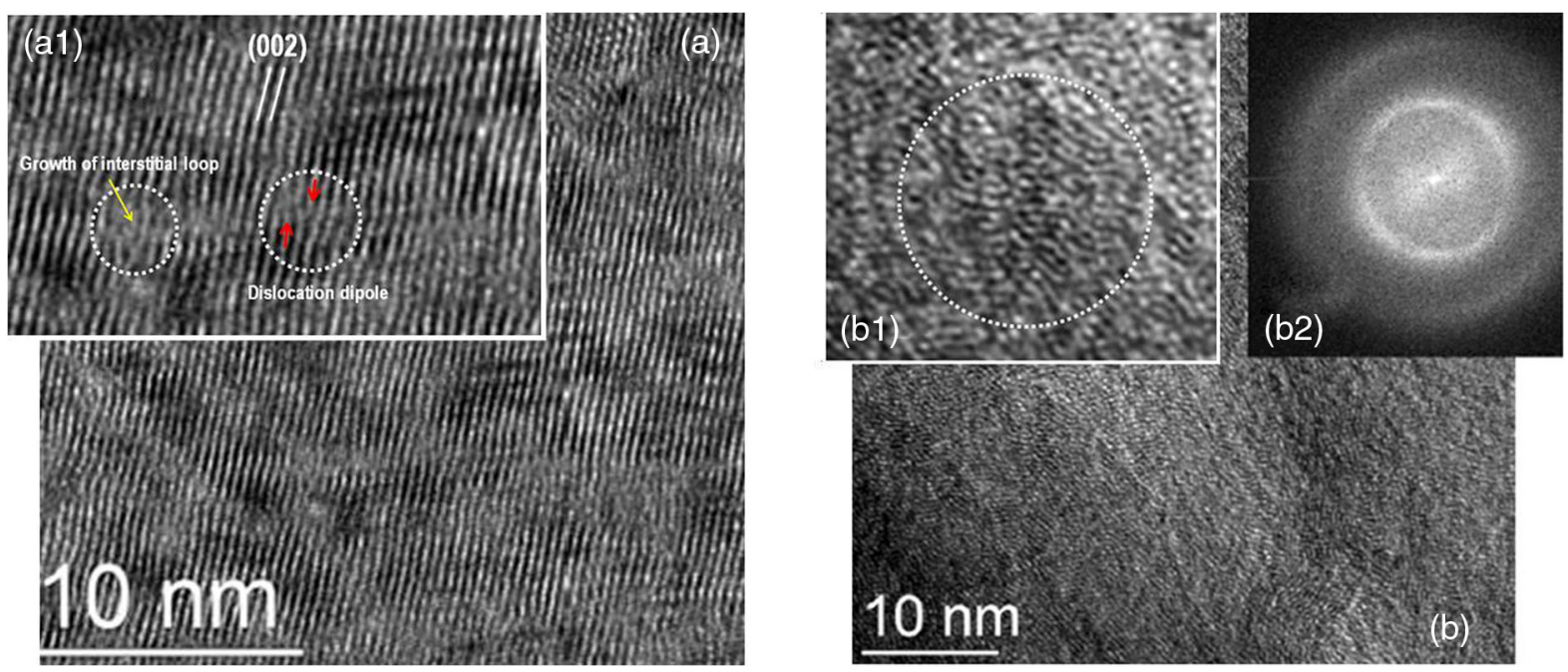

FIG. 20. (a) TEM micrograph at $3 \sigma$ showing lattice long-range order and pockets of dislocation dipoles, interstitial loops (a1) and (b) TEM micrograph within the beam $1 \sigma$ at the fracture surface subjected to $\sim 8.6 \times 10^{21} \mathrm{p} / \mathrm{cm}^{2}$ fluence with complete lattice fragmentation (b1) and diffused reflections (b2).

that the lattice structure is coherent with the basal planes well organized and visible. The lattice structure appears to only be moderately distorted or strained with localized pockets of disordered basal planes, dislocation dipoles, and growth of interstitial loops. X-ray diffraction results at similar distances from the beam center (i.e., $3 \sigma$ ) shown in Figs. 12 and 13 confirm the microscopy-based observations and reveal that the lattice structure [shown by several crystallographic reflections and primarily (002)] remains coherent and exhibits long-range order.

The state of the microstructure (lattice) changes dramatically near the beam center, as shown in Fig. 20(b). The lattice structure appears to be completely disordered, resulting in the fragmentation of the graphite crystallites into small grains leading to a nongraphitic structure. In Fig. 20(b), it is evident that the graphite basal planes lose their long-range order with the appearance of breaks and bends in the lattice, leading eventually to a disordered or randomized state [Figs. 20(b) and 20(b1)]. Planes are bent and twisted, while the diffractionlike image [Figs. 20(b) and 20(b2)] shows a diffused or broadened reflection similar to what is shown by XRD in Fig. 10(h). These findings, both at the beam center (within $1 \sigma$ ) and at its $3 \sigma$ tail, agree with the high-resolution x-ray diffraction findings. It should be emphasized that in the case of XRD the $0.5 \mathrm{~mm} \times 0.5 \mathrm{~mm} 67 \mathrm{keV}$ x-ray beam traversed the entire length $(20 \mathrm{~mm})$ of the target fin along the trace of the $120 \mathrm{GeV}$ proton beam that interacted with the target.

\section{SUMMARY AND CONCLUSION}

A comprehensive study based primarily on the diffraction of high-energy x-ray beams and augmented by electron microscopy as well as transport code simulations was undertaken to help unravel the effects of the $120 \mathrm{GeV}$ proton beam on the NT-02 graphite target of the NuMI experiment and identify the mechanism responsible for its failure. Experimental evidence of macroscopic and microscopic behavior of similar isotropic-grade graphites subjected to irradiation fluences that reached or surpassed the NT-02 target which was subjected to the unprecedented fluence level of $\sim 10^{22} \mathrm{~cm}^{-2}$ of protons compounded with spallation-generated neutrons was utilized. The primary objective was to pinpoint the driving failure mechanism and, in doing so, provide guidance to the target designs of future, multimegawatt power particle accelerator initiatives.

The x-ray diffraction study conducted using high-energy $x$ rays at the NSLS II XPD beam line revealed that at the extreme fluence approaching $10^{22} \mathrm{~cm}^{-2}$ irradiating particles (protons plus neutrons) the graphite material loses its long-range order and undergoes a complete crystalline-toamorphous transformation, diminishing its mechanical strength. The amorphization of the crystal lattice was corroborated by transmission electron microscopy. Such a finding is important to consider in the design of nextgeneration, multimegawatt targets in that there exists a fluence threshold beyond which the graphite's structural integrity is seriously compromised.

Macroscopic experiments on radiation-induced dimensional changes in the graphite (shrinkage and/or swelling) revealed the great sensitivity of the dimensional behavior with the irradiation temperature and the existence of two distinct temperature zones at about the $200^{\circ} \mathrm{C}$ level.

The nontypical swelling behavior of isotropic graphite, such as the NT-02 (POCO ZXF-5Q), results in excessive swelling at low irradiation temperatures even at a low dose.

As a result, aggravated by the tight $120 \mathrm{GeV}$ beam profile $(\sigma=1.1 \mathrm{~mm})$ which induces energy deposition and temperature profiles in the target of very high gradient, differential swelling or dimensional changes become a dominant factor in developing internal stresses in the target 
fins. These, combined with the "weakening" of the target fin midsection where the proton beam is intercepted, will result in the target failure observed. The resulting thermal stresses ( $231 \mathrm{MPa}$ compressive and $\sim 14 \mathrm{MPa}$ tensile) from the beam pulses totaling $\sim 1.4 \times 10^{7}$ (10 $\mu$ s pulse length with a repetition rate of $1.87 \mathrm{~s}$ ) may have contributed to fatigue even though the peak values are much lower than the unirradiated strengths of 170 and $75 \mathrm{MPa}$, respectively. It is believed that the large number of cycles experienced (and not necessarily the cyclic stress amplitude) combined with the irradiation-induced strength reduction contributed to either complete fracture or serious degradation of the target fins. The fact that fins located in the downstream half were also fractured, either in beam or were weakened and fractured during handling, is an indicator that the repetitious beam thermal shock, especially at this large number of pulses, had contributed to fin failure or fracture. Follow-up studies based on nanoindentation experiments on the fracture surfaces will help reveal the stress evolution or degradation over the critical target fin section and the role of fatigue in the observed fracture. Such results and assessment will be reported in a subsequent article.

Based on the failure mechanism assessment, it is recommended that graphite target designs for future multimegawatt accelerators consider (a) the observed fluence threshold that graphitic structures are capable of absorbing by appropriately increasing the size of the beam and (b) the sensitivity and variability of the microstructural and macrostructural behavior to the irradiation temperature allowing targets to operate at both higher and, if possible, uniform temperatures $\left(\gg 200^{\circ} \mathrm{C}\right)$.

\section{ACKNOWLEDGMENTS}

This document was prepared by the RaDIATE Collaboration using the resources of the Fermi National Accelerator Laboratory (Fermilab), a U.S. Department of Energy, Office of Science, HEP User Facility. Fermilab is managed by Fermi Research Alliance, LLC (FRA), acting under Contract No. DE-AC02-07CH11359. This research was supported by the DOE-NE Grant No. CT13BN040505. This research used resources of the Center for Functional Nanomaterials, which is a U.S. DOE Office of Science Facility, at Brookhaven National Laboratory under Contract No. DE-SC-0012704. This research used resources at XPD beam line of the National Synchrotron Light Source II, a U.S. Department of Energy (DOE) Office of Science User Facility operated for the DOE Office of Science by Brookhaven National Laboratory under Contract No. DE-SC-0012704.

[1] B. T. Kelly, The behavior of graphite under neutron irradiation, J. Vac. Sci. Technol. A 4, 1171 (1986).
[2] B. T. Kelly and J. E. Brocklehurst, High dose fast neutron irradiation of highly oriented pyrographite, Carbon 9, 783 (1971).

[3] B. T. Kelly, On the amorphization of graphite under neutron irradiation, J. Nucl. Mater. 172, 237 (1990).

[4] R. Taylor, R. G. Brown, K. Gilchrist, E. Hall, A. T. Hodds, B. T. Kelly, and F. Morris The mechanical properties of reactor graphite, Carbon 5, 519 (1967).

[5] N. Maruyama and M. Harayama, Neutron irradiation effect of thermal conductivity and dimensional change of graphite materials, J. Nucl. Mater. 195, 44 (1992).

[6] D. G. Schweitzer, Activation energy for single interstitials in neutron-irradiated graphite and the absolute rate of formation of displaced atoms, Phys. Rev. 128, 556 (1962).

[7] J.-P. Bonal, A. Kohyama, J. van der Laan, and L. L. Snead, Graphite, ceramics, and ceramic composites for hightemperature nuclear power systems, MRS Bull. 34, 28 (2009).

[8] W. Bollmann, Electron-microscopic observations on radiation damage in graphite, Philos. Mag. 5, 621 (1960).

[9] W. T. Eeles, Diffuse diffraction phenomena from neutronirradiated graphite single crystals, Acta Cryst. A24, 688 (1968).

[10] W. T. Eeles, Interpretation of the diffuse diffraction phenomena of neutron-irradiated graphite crystals, Philos. Mag. 32, 1273 (1975).

[11] A. Asthana, Y. Matsui, M. Yasuda, K. Kimoto, T. Iwata, and $\mathrm{K}$. Ohshima Investigations on the structural disordering of neutron-irradiated highly oriented pyrolytic graphite by x-ray diffraction and electron microscopy, J. Appl. Crystallogr. 38, 361 (2005).

[12] A. Burkholtz, Irradiation damage in graphite, CEN Report No. EUR 3056.e, 1966.

[13] B. J. Marsden, Irradiation damage in graphite due to fast neutrons in fission and fusion systems, Report No. IAEATECDOC-1154, 2000.

[14] B. J. Marsden, M. Haverty, W. Bodel, G. N. Hall, A. N. Jones, P. M. Mummery, and M. Treifi, Dimensional change, irradiation creep and thermal/mechanical property changes in nuclear graphite, Int. Mater. Rev. 61, 155 (2016).

[15] T. Saito and Y. Sasaki, Effect of high-temperature neutron irradiation on the crystallites of graphite materials, Carbon 18, 407 (1980).

[16] J. Eapen et al., Early damage mechanisms in nuclear grade graphite under irradiation, Mater. Res. Lett. 2, 43 (2014).

[17] L. L. Snead, T. D. Burchell, and Y. Katoh, Swelling of nuclear graphite and high-quality carbon fiber composite under very high irradiation temperature, J. Nucl. Mater. 381, 55 (2008).

[18] J. Gittus, Creep, Viscoelasticity and Creep Fracture in Solids (Wiley, New York, 1975).

[19] C. Hubert et al., High resolution synchrotron x-ray diffraction of swift heavy ion irradiated graphite, GSI Scientific Report No. PNI-INHOUSE-EXP-39, 2012.

[20] N. Simos, H. G. Kirk, P. Thieberger, H. Ludewig, J. O. Conor, L. Mausner, P.-T. Trung, K. T. McDonald, K. Yoshimura, and J. R. J. Bennett, Irradiation damage studies of high power accelerator materials, J. Nucl. Mater. 377, 41 (2008). 
[21] N. Simos et al., Multi-MW accelerator target material properties under proton irradiation at Brookhaven National Laboratory linear isotope producer, Phys. Rev. Accel. Beams 21, 053001 (2018).

[22] N. Simos, P. Nocera, Z. Zhong, R. Zwaska, N. Mokhov, J. Misek, K. Ammigan, P. Hurh, and Z. Kotsina, Proton irradiated graphite grades for a long baseline neutrino facility experiment, Phys. Rev. Accel. Beams 20, 071002 (2017).

[23] N. Simos et al., Radiation damage and thermal shock response of carbon-fiber-reinforced materials to intense high-energy proton beams, Phys. Rev. Accel. Beams 19, 111002 (2016).

[24] N. Simos et al., Long Baseline Neutrino Experiment (LBNE) target material radiation damage from energetic protons of the Brookhaven Linear Isotope Production (BLIP) facility, Report No. BNL 111826-2016-IR.
[25] T. T. Böhlen, F. Cerutti, M. P. W. Chin, A. Fassò, A. Ferrari, P. G. Ortega, A. Mairani, P. R. Sala, G. Smirnov, and V. Vlachoudis, The FLUKA code: Developments, and challenges for high energy, and medical applications, Nucl. Data Sheets 120, 211 (2014).

[26] A. Ferrari, P. R. Sala, A. Fasso, and J. Ranft, FlukA: A multi-particle transport code, Report No. CERN-2005-10, INFN/TC_05/11, SLAC-R-773, 2005.

[27] N. V. Mokhov, The MARS code system user's guide, Report No. Fermilab-FN-628, 1995; N. V. Mokhov and S. I. Striganov, MARS15 overview, AIP Conf. Proc. 896, 50 (2007); N. V. Mokhov et al., Prog. Nucl. Sci. Technol. 4, 496 (2014), DOI: 10.15669/pnst.4.496, http://www-ap .fnal.gov/MARS/.

[28] LS-DYNA, version 9.71, Livermore Software Technology Corp. (LSTC), 2007. 